\title{
A High-Order Cut-Cell Method for Numerical Simulation of Hypersonic-Boundary Transition with Arbitrary Surface Roughness
}

\author{
Le Duan ${ }^{*}$, Xiaowen Wang ${ }^{\dagger}$ and Xiaolin Zhong ${ }^{\ddagger}$ \\ University of California, Los Angeles, California 90095
}

\begin{abstract}
Hypersonic boundary-layer transition can be affected significantly by surface roughness. Many important mechanisms which involve transition induced by arbitrary roughness are not well understood. In this paper, we propose a new high-order cut cell method which combined the non-uniform finite difference method for discrete points near the curvilinear boundary and shock-fitting method for the bow shock. The receptivity process induced by interaction of Mach 5.92 flow over flat plate under the combination effect of twodimensional surface roughness and blow-suction is investigated. Both steady state solutions and unsteady solutions have been obtained by using the new method. For steady flow with roughness, there is significant change inside the boundary layer with flow separation before and after the roughness element. For unsteady flow, the results for flow instability induced by both blow-suction slot and roughness are obtained and analyzed by Linear Stability Theory (LST). These results show that the roughness element with height to be half the boundary layer thickness may delay the hypersonic transition.
\end{abstract}

\section{Introduction}

Understanding the roughness induced laminar-turbulence transition of boundary layer serves as a critical design issue for hypersonic vehicles. Transition can have a first order influence on their body lift and drag, stability and control and heat transfer property. For example, swept wings are used for most commercial and military aircraft. It has been know that the aerodynamics parameter may vary substantially after transition. Thus to understand the fundamental instability mechanisms in swept-wing flows is crucial considering its board application in aerospace design [1]. Another application of studying roughness induced transition is for the design of thermal heat protection system of hypersonic vehicles. For a reentry vehicle entering earth's atmosphere, it initially experiences a heating environment associated with a laminar boundary layer. Eventually with the attitude decreasing, the vehicle surface become rougher and the boundary layer become turbulent and the heating rate at the surface can be increased by a factor of four or more [2]. Thus the ability to understand and predict the roughness induced transition plays an essential role in thermal protection system (TPS) design process. However, surface roughness, especially arbitrary roughness induced laminar-turbulence transition in hypersonic boundary layers is still poorly understood due to the limitation in experimental facilities and numerical methods [4].

For problems having complex computational geometry, as might occur in the transition problems induced by isolated/distributed roughness, the use of body fitted curvilinear grids could prove to be very difficult due to natural complexities in grid generation, especially for three-dimensional arbitrary domain. Consequently, one approach for overcoming this difficulty is to use a cut cell method, which is easy in generating and implementing numerical schemes. Cut cell methods can take full advantage of fast computer architectures like vector or parallel computers and could serve as a very flexible method for simulating flow around complex geometries. It is first used for solving the equations of transonic potential flow by Purvis and Burkhalter [15]. Then it is further developed to calculate steady compressible flows by Clarke et al. [16].

\footnotetext{
* Graduate Research Assistant, Department of Mechanical\&Aerospace Engineering, Member, AIAA

${ }^{\dagger}$ Research Scientist, Department of Mechanical\&Aerospace Engineering, Member, AIAA

¥ Professor, Department of Mechanical\&Aerospace Engineering, Associate Fellow, AIAA
} 
Without considering the stability and accuracy issues, various cut cell methods have been developed and can be modified to solve problems with arbitrary geometry [32-38]. One of the most popular methods is Peskin's immersed boundary method (IBM) [35]. In his simulation of blood flow in the heart, the force induced by heart valve is represented by a discrete delta function. These forces are added in to the Navier-Stocks equations to reflect the immersed boundary between two phase flows where the resulting equations are discretized by standard finite difference methods in a fixed Cartesian girds. However, since the immersed boundary method uses the discrete delta function approach, it smears out the sharp interface to a thickness of order of the mesh width. Thus the immersed boundary method is proved to be only first order accurate, even though a high-order approximation can be achieved for smooth boundary problems.

There is another kind of cut cell methods referring to the 'sharp interface' method [18, 32], where the jump condition across the sharp interface is determined and incorporated into the numerical scheme explicitly. As one of the sharp interface Cartesian girds methods, Udaykumar et al. [32-34] use finite volume method and finite difference methodology to solve the incompressible flow over complex and moving geometries. Their solver employs a second-order accurate central difference method for spatial discretization and an explicit-implicit fractional step scheme for time advancement, which allows a simulation of unsteady viscous incompressible flow with complex immersed boundary conditions. A one side bilinear interpolation is applied to calculate the accurate flow condition in the grids cut by boundary. Colella et al. [18-20] develop a sharp cut cell method in a manor that discretizating the conservative equations on a finite volume and factional cells with irregular boundary. The stability and local conservation is achieved by redistributing the differences of the mass increment from the irregular cell to the adjacent regular cells.

As an alternative to sharp cut cell method, Fedkiw et al. [25, 36, 37] develop a ghost fluid method for solving equation in arbitrary domain. The basic idea is to extrapolate variables on one side of the boundary into the ghost cell in other side, which is located inside the solid boundary. In [25] Gibou and Fedkiw improve their method to fourth-order accuracy with finite difference discretization for solving the Laplace and heat equations in irregular domain.

Recently a new cut cell approach referring to immersed interface method is developed and widely used in simulating multi-phase flow [6-12,37-40]. This method can achieve second or higher order global accuracy by incorporating jump conditions for variables and their derivatives given at the interface. This method is first used by Leveque and Li to solve an incompressible Navier-Stokes equation [37]. Leveque et al. then extend their immersed interface method to solve Navier-Stokes equation with surface tension [38]. Wiegmann and Bube [39, 40] develop an explicit jump immersed interface method for special cases where the explicit jump condition of variables and their high-order derivatives are known. This method can only achieve arbitrary high-order accuracy with the premise that the corresponding high-order derivatives of jump condition can be analytically derived. Thus the number of viable partial differential equations satisfying such explicit jump condition restriction is very limited.

Among those applications, a problem referring to restriction on time step would arise when we are trying to implement high-order numerical scheme in the relatively small-sized irregular cell. This constriction which refers to "small cell problem", would significantly delay the temporal advancement, as well as increasing the computational cost. Many innovative methods are developed to resolve the small cell problem. Berger and Leveque [17] use rotating box method. Colella et al. [18-20] use flux-redistribution procedures, Quirk [21] and Shyy [22] use cell merging method to get numerical stability. However, all the cut cell methods with different boundary treatments is proved to be only first or second order, thus are not sufficient in numerically simulating laminar-turbulent boundary transitions. Shyy et al. [24] extend his previous second-order method to fourth-order with merging cell approach. But the procedure for reconstruction flux is relatively expensive thus slowing down the computational efficiency. Fedkiw et al. [26] presented a stable fourth-order finite difference method for solving the Laplace equation on an irregular domain. A good rule of thumb for removing small cell restriction proposed in [26] is that the interpolation should be shift to be centered one grid points left or right if the interpolation stencil involves point considered to be relatively close to the boundary.

In our previous paper [45], a new stable high order cut cell method is developed to simulate Mach 5.92 flow over flat plate with arbitrary surface roughness. The computational result shows that due to the existence of surface roughness, the hypersonic flow over flat plate is compressed and a weak oblique shock is formed in front the roughness and then an expansion wave is followed. The results also show that there is a significant change of flow profiles inside the boundary layer in the downstream of surface roughness. In this paper, we consecutively present the computational results of roughness induced transition in flat plate for unsteady flow with a high-order cut-cell method. A high order (up to fifth-order) uniform finite difference scheme is used in simulating later zones behind the roughness. The bow shock generated from the leading edge of the flat plate will be treated as a boundary condition and discretized based on Zhong's [41] fifth-order finite difference flux split method and shock fitting 
method. The unsteady flow over flat plate is excited by a blow-suction composed of 15 different frequencies mounted at $0.030 \mathrm{~m}$ downstream from the leading edge. The unsteady state solution is obtained with a surface roughness placed on the flat plate $0.185 \mathrm{~m}$ downstream with height to be half of boundary-layer thickness.

\section{Governing Equations}

The governing equations for the numerical simulation of hypersonic boundary layer transition are the threedimensional Navier-Stokes equations. We assume that we are dealing with Newtonian fluids with perfect gas assumption and isothermal wall condition. The governing equations can be written in the following conservationlaw form in the Cartesian coordinates.

$$
\frac{\partial U}{\partial t}+\frac{\partial F_{j}}{\partial x_{j}}+\frac{\partial F_{v j}}{\partial x_{j}}=0
$$

where $U, F_{j}$ and $F_{v j}$ are the vectors of flow variables, convective flux, and viscous flux in the $j$ th spatial direction respectively, i.e.,

$$
\begin{gathered}
U=\left\{\rho, \rho u_{1}, \rho u_{2}, \rho u_{3}, e\right\} \\
F_{j}=\left\{\begin{array}{l}
\rho u_{j} \\
\rho u_{j 1} u_{j}+p \delta_{1 j} \\
\rho u_{j 2} u_{j}+p \delta_{1 j} \\
\rho u_{j 3} u_{j}+p \delta_{1 j} \\
(e+p) u_{j}
\end{array}\right\} \\
F_{v j}=\left\{\begin{array}{l}
0 \\
\tau_{1 j} \\
\tau_{2 j} \\
\tau_{3 j} \\
\tau_{j k} u_{k}-q_{j}
\end{array}\right\}
\end{gathered}
$$

In this paper, only perfect-gas hypersonic flow is considered, i.e.,

$$
\begin{gathered}
p=\rho R T \\
e=\rho\left(C_{v} T+\frac{1}{2} u_{k} u_{k}\right) \\
\tau_{i j}=\mu\left(\frac{\partial u_{i}}{\partial x_{j}}+\frac{\partial u_{j}}{\partial x_{i}}\right)+\delta_{i j} \lambda \frac{\partial u_{k}}{\partial x_{k}} \\
q_{j}=-k \frac{\partial T}{\partial x_{j}}
\end{gathered}
$$

where $R$ is the gas constant. The specific heats $C_{v}$ are assumed to be constants with a given ratio of specific heats $\gamma$. The viscosity coefficient $\mu$ can be calculated by Sutherland's law in the form: 


$$
\mu=\mu_{r}\left(\frac{T}{T_{0}}\right)^{3 / 2} \frac{T_{0}+T_{S}}{T+T_{S}}
$$

Where $\mu_{r}=1.7894 \times 10^{-5} \mathrm{Ns} / \mathrm{m}^{2}, T_{0}=288.0 \mathrm{~K}, T_{s}=110.33 \mathrm{~K}$ and $\lambda$ is assumed to be $-2 / 3 \mu$. The heat conductivity coefficient $\gamma$ can be computed through a constant Prantl number Pr.

\section{Numerical Methods}

\section{A. Discretization in Space - High-Order Finite Difference and Shock-Fitting Method}

To satisfy the accuracy requirement for capturing small disturbances inside the boundary layer for receptivity and transition simulation, a three-dimensional fifth-order shock-fitting method developed by Zhong is used to compute the flow field bounded by the bow shock and wall surface. In the discretization of the Navier-Stokes equations, spatial derivatives in the streamwise $(\xi)$ and wall-normal $(\eta)$ directions are modeled by a fifth-order finite difference scheme. The flow variables behind the shock are determined by the Rankine-Hugoniot relations across the shock and a characteristic compatibility equation behind the shock.

Before implementing the proposed numerical scheme, the physical domain should be transformed into a cub computational domain bounded by oscillating shock and flat plate boundary. Under the computational coordinate system, the body fitted girds are represented by a curvilinear three-dimensional coordinates $(\xi, \eta, \varsigma, \tau)$ along the gird lines. The unsteady movement of the bow shock is treated as the computational upper bound at $\eta=\eta_{\max }$, which is time dependent. The other gird line $\xi=$ const $\varsigma=$ const remains stationary while computing. The coordinate transformation is defined by:

$$
\left\{\begin{array} { l } 
{ \xi = \xi ( x , y , z , t ) } \\
{ \eta = \eta ( x , y , z , t ) } \\
{ \varsigma = \varsigma ( x , y , z , t ) } \\
{ \tau = t }
\end{array} \longrightarrow \left\{\begin{array}{l}
x=x(\xi, \eta, \varsigma, \tau) \\
y=y(\xi, \eta, \varsigma, \tau) \\
z=z(\xi, \eta, \varsigma, \tau) \\
t=\tau
\end{array}\right.\right.
$$

where $(x, y, z, t)$ is defined under Cartesian coordinate system.

The optimal formation of transformation formula is determined by the specific physical model. For viscous flow simulation, two steps of mapping procedure could be implemented to obtain better resolution inside the viscous layer. As shown in Fig 1. The first step is involving transforming the entire physical domain under Cartesian coordinates into a cub defined on $[0,1] \times[0,1] \times[0,1]$ under an intermediate coordinate space. The relation is defined as:

$$
\left\{\begin{array}{l}
X=\frac{x-x_{\text {strart }}}{L} \\
Y=\frac{y}{H(x, y)} \\
Z=z
\end{array}\right.
$$

Where $(X, Y, Z)$ is defined under the intermediate coordinate system. $L$ is the streamwise length of the flat plate in current physical domain. $x_{\text {start }}$ is the physical streamwise coordinate of starting point of computation. The distance along $\eta$ between the solid wall and bow shock is referred to $H$.

In the second step of transformation, the intermediate plane $(X, Y, Z)$ is mapped into the computational domain $(\xi, \varsigma, \tau)$ in order to cluster more girds into the viscous layer on the flat plate. In present study, an exponential stretch function is employed as following, 


$$
\left\{\begin{array}{l}
X=\xi \\
Y=-\frac{\left(\frac{\beta+1}{\beta-1}\right)^{(1-\eta)}-1}{\left(\frac{\beta+1}{\beta-1}\right)^{(1-\eta)}+1} \beta \\
Z=\zeta
\end{array}\right.
$$

where $\beta$ is the stretching parameter with value set to be 1.0 in present simulation. The combination of the two steps leads to equation (10) .

Equation (10) can be substituted into the governing equation (1), which may lead to a system of transformed equations in the computational domain $(\xi, \eta, \zeta, \tau)$ as

$$
\frac{1}{J} \frac{\partial U}{\partial \tau}+\frac{\partial E^{\prime}}{\partial \xi}+\frac{\partial F^{\prime}}{\partial \eta}+\frac{\partial G^{\prime}}{\partial \zeta}+\frac{\partial E_{v}^{\prime}}{\partial \xi}+\frac{\partial F_{v}^{\prime}}{\partial \eta}+\frac{\partial G_{v}^{\prime}}{\partial \zeta}=0
$$

where

$$
\begin{array}{r}
E^{\prime}=\frac{F_{1} \xi_{x}+F_{2} \xi_{y}+F_{3} \xi_{z}}{J} \\
F^{\prime}=\frac{F_{1} \eta_{x}+F_{2} \eta_{y}+F_{3} \eta_{z}}{J} \\
G^{\prime}=\frac{F_{1} \zeta_{x}+F_{2} \zeta_{y}+F_{3} \zeta_{z}}{J} \\
E_{v}{ }^{\prime}=\frac{F_{v 1} \xi_{x}+F_{v 2} \xi_{y}+F_{v 3} \xi_{z}}{J} \\
F_{v}{ }^{\prime}=\frac{F_{v 1} \eta_{x}+F_{v 2} \eta_{y}+F_{v 3} \eta_{z}}{J} \\
G_{v}{ }^{\prime}=\frac{F_{v 1} \zeta_{x}+F_{v 2} \zeta_{y}+F_{v 3} \zeta_{z}}{J}
\end{array}
$$

Where $\xi_{x}, \xi_{y}, \xi_{z}, \eta_{x}, \eta_{y}, \eta_{z}, \varsigma_{x}, \varsigma_{y}, \varsigma_{z}$ are transformation metrics and $J$ is Jacobean matrix of coordination transformation defined by

$$
J=\frac{\partial(\xi, \eta, \varsigma)}{\partial(x, y, z)}
$$

The grids transformation metrics and the Jacobean matrix could be calculated through applying derivative chain rule into the relation of equation (10). With the coordinate transformation, uniform schemes could be applied in the computational domain where in reality the grids distribution in physical domain is non-uniform.

In this paper, the shock interface is treated as one of the boundary of the domain. A fifth order shock-fitting method developed by Zhong is used to determine the physical boundary condition. The flow variables behind the shock are determined by the Rankine-Hugonion condition and characteristic compatibility relations behind the shock. The velocity of shock front and acceleration of shock front could be written as the relation,

$$
\left\{\begin{array}{l}
\frac{\partial H}{\partial \tau}=H_{\tau} \\
H_{\tau \tau}=H_{\tau \tau}\left(\xi, \zeta, H, \tau, P_{\infty}, M a_{\infty}, \rho_{\infty}\right)
\end{array}\right.
$$


$H_{\tau \tau}\left(\xi, \zeta, H, \tau, P_{\infty}, M a_{\infty}, \rho_{\infty}\right)$ is a relation determined by time and the freestream side of physical boundary condition $P_{\infty}, M a_{\infty}, \rho_{\infty}$. With time advancing in computation, the shock shape as well as the physical domain is updated in each simulation step according to equation (20). The details about derivation of the shock fitting formulas and numerical methods can be found in [41].

The transformed equation (13) is discretized by a high-order uniform finite difference method. In the inviscid flux term, the Jacobian matrix contains both positive and negative eigenvalues, thus for pursuing more stable computation, two sets of schemes are employed to calculate viscous and inviscid flux term separately. A fifth-order upwind scheme combined with a local Lax-Friedrichs flux split scheme is used for discretizating the inviscid flux derivatives, while a six order central scheme is used to discrete the viscous flux derivatives.

The inviscid flux term in equation (13) can be divided into two parts with pure negative and position values as

$$
F^{\prime}=F^{\prime^{+}}+F^{\prime^{-}}
$$

To obtain equation (21), a general method is to choose a sufficient large parameter $\lambda$, like

$$
\lambda=\frac{|\nabla \eta|}{J}\left(\sqrt{(\varepsilon c)^{2}+u^{2}+c}\right.
$$

where $c$ is the speed of sound, $\varepsilon$ is a small variable which introduce smoothness of the flux splitting, and $u$ can be calculated by

$$
u^{\prime}=\frac{\eta_{x} u+\eta_{y} v+\eta_{z} w+\eta_{t}}{|\nabla \eta|}
$$

$\lambda$ could be chosen to be larger than any of the absolute eigenvalue of $F^{\prime}$. Then $F^{\prime^{+}}, F^{\prime^{-}}$could be defined as

$$
\left\{\begin{array}{l}
F^{\prime^{-}}=\frac{1}{2}\left(F^{\prime}-\lambda U\right) \\
F^{\prime^{+}}=\frac{1}{2}\left(F^{\prime}+\lambda U\right)
\end{array}\right.
$$

Since $F^{\prime-}$ and $F^{\prime+}$ contains only pure negative and positive value respectively, the fifth-order explicit upwind scheme could be used to discretize their derivatives in order to improve the overall computational stability. A difference equation for first order derivatives could be written as

$$
\frac{\partial F^{\prime^{+}}}{\partial \xi}=\frac{1}{\Delta h} \sum_{k=-3}^{3} a_{i+k} F^{\prime^{+}}{ }_{i+k}-\frac{\alpha}{6 !}\left(\frac{\partial F^{\prime+}}{\partial \xi}\right)_{i}^{6}+\ldots \ldots
$$

where $\Delta h$ is the size of spatial grids. The coefficients can be calculated by using Taylor expansion as follows:

$$
\left\{\begin{array}{l}
a_{i}=\frac{1}{b}\left(-\frac{5}{3} \alpha\right) \\
a_{i \pm 1}=\frac{1}{b}\left( \pm 45+\frac{5}{4} \alpha\right) \\
a_{i \pm 2}=\frac{1}{b}\left(\mp 9-\frac{1}{2} \alpha\right) \\
a_{i \pm 3}=\frac{1}{b}\left( \pm 1+\frac{1}{12} \alpha\right) \quad b=60
\end{array}\right.
$$


When $\alpha<0$ the scheme is upwind and when $\alpha=0$, the scheme is sixth-order central difference, which is used to discrete the viscous terms. Combined with cut cell method for boundary treatment, the spatial discretization towards equation (13) leads a system of ordinary partial equation. For steady problem, a first-order Euler scheme is used to discrete the system of ODEs, which in turn lead to a system of linear equations. Final solution can be obtained through temporal integration. The stability analysis for the high-order finite difference method can be found in [41].

\section{B. Treatment of Fluid-Solid Boundary - Cut Cell Method}

In this section, we mainly focus on deriving the cut cell method in the context of two-dimensional simulation. The method can be extended to three dimensions naturally.

After the coordinate transformation as defined in equation (13), a set of body-fitted girds can be generated in the two-dimensional square computational domain without roughness. Some of the Cartesian cells may cut by the roughness boundary which then may lead to irregular Cartesian cells. It is worthy noting that the roughness geometry should be transformed into be square space following the equation (10) while computing the metrics associated with roughness boundary.

Four different kinds of grids located in the vertex of those Cartesian cells are defined and different numerical algorithms are implemented. They are regular point, irregular point, boundary point, and dropped point as shown in Fig. 2. The intersections of roughness interface and grids lines are defined as boundary points. The rest points produced by intersections of grids lines themselves are termed as regular points, irregular points and dropped points respectively. The criteria for distinguishing those are depending on their minimum distances to the solid wall. If the grids points adjacent to a boundary point with a distance smaller than a pre-specified critical ratio $\Theta$ in wall-normal or streamwise direction, they are defined as dropped points and are took off from the gird stencil in corresponding direction, which is terms as "dropped direction". Please be aware that the points are abandoned in the "dropped direction" only, they may be included in difference stencils in directions that are not defined as "dropped". In the third and fifth order simulation, the critical ratio $\Theta$ is selected to be $0.5 \Delta h$ and $0.9 \Delta h$ where desirable stable solution can be obtained as shown in following sections. For those points whose finite difference stencil may include boundary points, they are defined as irregular points. Then the left points which cover the entire domain are defined as regular points since they are relatively far away from boundary points, where a standard uniform finite difference approach can be applied.

The flux terms in regular points are computed by upwind finite difference scheme introduced in previous section A, which might take up to a seven point stencil. To calculate the flux in irregular points, a non-uniform finite difference method, whose stencil is consisted of regular, irregular and boundary points, is developed. It is worthy noting the points marked by red dot in Fig. 2 are dropped, they should not be included into non-uniform or uniform stencils for computing flux terms in "dropped direction". The main purpose of defining dropped point is to avoid the small cell problem introduced in section one. After abandoning the dropped points, the small segment in a nonuniform difference stencil between the dropped point and irregular point are enforced to merge into a larger segment which connects the nearby boundary point and irregular point directly. This kind of grids definition and segment merging approach ensure that distance between two adjacent grids in the difference stencil to be large enough thus preventing from the deterioration of corresponding high-order interpolation and finite difference method while computing the hyperbolic part of equation (13).

The steps to derive the numerical method for both irregular and boundary points and to implement our cut cell method are described as follows.

\section{Viscous term in irregular points}

In previous section we employ up to fifth-order accuracy finite difference scheme for regular points discretization. To maintain fifth-order global accuracy for viscous term, a fourth-order accuracy non-uniform scheme with five grids stencil should be used for discretizating irregular points near the boundary in computational domain as shown in Fig 3. By the definition of irregular points, their finite difference stencil must contain boundary points. The formulation of non-uniform difference equation with respect to viscous term can be written as 


$$
\frac{\partial F_{v, i}{ }^{\prime}}{\partial \xi}=\frac{1}{\Delta h} \sum_{k=1}^{5} a_{i k}(\Delta h, \theta) F_{v, k}{ }^{\prime}-\frac{C(\Delta h)^{4}}{5 !}\left(\frac{\partial F_{v^{\prime}}}{\partial \xi}\right)_{i}^{5} \quad \text { for } i=2,3,4
$$

The subscripts of coefficient $a_{i k}$ stand for the $k t h$ coefficient for the $i t h$ irregular point as defined in Fig $3 . a_{i k}$ is function of $(\Delta h, \theta)$ with $\Delta h$ and $\theta$ are the uniform and non-uniform girds space after removing the dropped points. $C$ is a constant.

The coefficients of difference equation for overall irregular points can be ranked as a $(3 \times 5)$ matrix with element $a_{i k}$, Every entry for the coefficient matrix can be derived through taking derivatives towards Lagrange interpolation polynomials generated from given stencil. For each irregular point, the Lagrange interpolation polynomial with five points stencil can be written as

$$
F_{v}^{\prime}(\xi)=\sum_{s i=1}^{5}\left(\prod_{s j=1, s j \neq s i}^{5} \frac{\xi-\xi_{s j}}{\xi_{s i}-\xi_{s j}}\right) F_{v, s i}
$$

Differentiating equation (28) once with respect to $\xi$,

$$
\partial \frac{F_{\nu}^{\prime}(\xi)}{\partial \xi}=\sum_{s i=1}^{5}\left(\sum_{s k=1, s k \neq i}^{5}\left(\prod_{s j=1, s j \neq s i, s j \neq s k}^{5} \frac{\xi-\xi_{s j}}{\xi_{s i}-\xi_{s j}}\right) \frac{1}{\xi_{s i}-\xi_{s k}}\right) F_{v, s i}{ }^{\prime}
$$

The coefficient $a_{i k}$ can be calculated by substituting $\xi=\xi_{i}$ as

$$
a_{i k}=\sum_{s k=1, s k \neq k}^{5}\left(\prod_{s j=1, s j \neq k, s j \neq s k}^{5} \frac{\xi_{i}-\xi_{s j}}{\xi_{k}-\xi_{s j}}\right) \frac{1}{\xi_{k}-\xi_{s k}} \text { for } i=2,3,4 \text { and } k=1,2 . .5
$$

From equation (30) we can infer that there is a one to one mapping relation between the set of coefficients and non-uniform grids space $\theta$ with fixed $\Delta h$ at each time step. The coefficients matrix for viscous term discretization can be derived explicitly and written as,

$$
A=\left[\begin{array}{ccccc}
\frac{-6}{\sigma(1+\sigma)(2+\sigma)(3+\sigma)} & \frac{-11 \sigma+6}{6 \sigma} & \frac{3 \sigma}{1+\sigma} & \frac{-3 \sigma}{2+\sigma} & \frac{\sigma}{3(3+\sigma)} \\
\frac{2}{\sigma(1+\sigma)(2+\sigma)(3+\sigma)} & \frac{-2(\sigma+1)}{6 \sigma} & \frac{-\sigma+1}{2(1+\sigma)} & \frac{1+\sigma}{2+\sigma} & \frac{-(1+\sigma)}{6(3+\sigma)} \\
\frac{-2}{\sigma(1+\sigma)(2+\sigma)(3+\sigma)} & \frac{\sigma+2}{6 \sigma} & \frac{\sigma+2}{1+\sigma} & \frac{4+\sigma}{2+\sigma} & \frac{2+\sigma}{6(3+\sigma)}
\end{array}\right]_{3 \times 5}
$$

Where $\sigma=\frac{\theta}{\Delta h}$. For the third order simulation, the critical ratio $\Theta=0.5 \Delta h$ such that $\sigma \geq 0.5$.

In terms of obtaining difference coefficients, the Lagrange interpolation approach is preferable to Taylor expansion approach since solving system of linear equation is relatively more expensive while performing numerical computing. Further more, the condition number of the linear equation system derived from Taylor expansion approach may be close to zero, which would in turn induce large round off numerical error.

It is important to realize that the grids metrics in computational domain bounded by the moving shock vary in each step. Thus we need to recalculate the finite difference coefficients for corresponding irregular point in each iteration step. In our simulation, the coefficients matrix (31) is stored in the computer memory and computed explicitly for implementing finite difference scheme in each direction. 
I need to restate that all the implementation of finite difference scheme must be conducted in the circumstance that all the dropped points have been removed from the computational domain in "dropped direction". Thus size of non-uniform grids space $\theta$ may be larger than the normal grids size $\Delta h$.

\section{Inviscid term in irregular points}

To be consistent with the aforementioned uniform finite difference method for regular points, a non-uniform high-order upwind scheme is developed to disretize the positive flux variable $F^{1+}$ and negative inviscid flux variable $F^{t^{-}}$as defined in equation (24). Being different with upwind schemes for computing viscous variables, at each given order of accuracy there are several sets of continuous grids stencils available to be chosen for approximating the derivatives of both negative and positive inviscid flux terms provided that these schemes are "upwind" and are third order or higher. The qualifying stencils may or may not include the boundary point. Thus the flexibility of selecting finite difference stencil for every irregular point may lead to many combinations of boundary closure upwind schemes possessing different stability characteristics for the overall simulation.

In terms of releasing time step restrictions, the trade off between the total number of boundary points contained in and width of the stencil of all irregular points implies that a potential optimal combination of boundary closure scheme may exist. One of the most stable (least temporal restriction) third-order non-uniform upwind finite difference schemes in irregular points is shown in Fig 4. For irregular point indexed by $i=3$, the stencil of upwind scheme is point set $\left(\xi_{2}, \xi_{3}, \xi_{4}, \xi_{5}\right)$ for positive flux $F^{{ }^{+}},\left(\xi_{1}, \xi_{2}, \xi_{3}, \xi_{4}\right)$ for negative flux $F^{{ }^{\prime}}$. For irregular point indexed by $i=2$, the stencil of upwind scheme is $\left(\xi_{2}, \xi_{3}, \xi_{4}\right)$ for positive flux $F^{\prime+},\left(\xi_{1}, \xi_{2}, \xi_{3}\right)$ for negative flux $F^{\prime-}$. The coefficients for each upwind scheme can be calculated following equation (28) and standard Lagrange differentiate approach.

The discretization scheme for inviscid term discretization can be written as

$$
\begin{gathered}
\frac{\partial F_{i}^{+}}{\partial \xi}=\frac{1}{\Delta h} \sum_{k=1}^{5} b^{+}{ }_{i k} F_{k}^{+} \quad \text { for } i=2,3 \\
\frac{\partial F_{i}^{-}}{\partial \xi}=\frac{1}{\Delta h} \sum_{k=1}^{5} b_{i k}^{-}(\sigma) F_{k}^{-} \quad \text { for } i=2,3
\end{gathered}
$$

The coefficients for each upwind scheme can be calculated following equation (30) and can be written in a matrix form

$$
\begin{gathered}
B^{+}=\left[\begin{array}{rrrrr}
0 & -\frac{3}{2} & 2 & -\frac{1}{2} & 0 \\
0 & -\frac{1}{3} & -\frac{1}{2} & 1 & -\frac{1}{6}
\end{array}\right]_{2 \times 5} \\
B^{-}=\left[\begin{array}{lllll}
-\frac{1}{\sigma(1+\sigma)} & \frac{(1-\sigma)}{\sigma} & \frac{\sigma}{(1+\sigma)} & 0 & 0 \\
\frac{1}{\sigma(1+\sigma)(2+\sigma)} & -\frac{\sigma+1}{2 \sigma} & \frac{1}{(1+\sigma)} & \frac{1+\sigma}{2(2+\sigma)} & 0
\end{array}\right]_{2 \times 5}
\end{gathered}
$$

The coefficients matrixes $B^{+}$and $B^{-}$are stored in the computer memory and computed explicitly for difference scheme at each iteration step.

\section{Boundary points}

High-order finite difference method requires numerical boundary conditions with comparable order to maintain global accuracy. To maintain the third-order global accuracy of upwind schemes developed in last section, it is 
desirable to have at least second-order accuracy scheme at boundary. In the solid interface imposing the non-slip, non-through and isothermal wall condition, the unique unknown physical variable in equation (10) is pressure which should be part of the solution.

Assume that the zero point for extrapolation in computational domain is originated at the irregular point at $\xi=\xi_{2}$ as in Fig. 3 Linear and forth order non-uniform extrapolation formulas can be constructed as follows,

Linear second-order extrapolation: take interpolant $\tilde{P}(\xi)=a \xi+b$ with conditions

$$
\left\{\begin{array}{l}
\tilde{P}(0)=P\left(\xi_{2}\right) \\
\tilde{P}(\Delta h)=P\left(\xi_{3}\right)
\end{array}\right.
$$

Fourth-order extrapolation: take interpolant $\tilde{P}(\xi)=a \xi^{3}+b \xi^{2}+c \xi+d$ with conditions

$$
\left\{\begin{array}{l}
\tilde{P}(0)=P\left(\xi_{2}\right) \\
\widetilde{P}(\Delta h)=P\left(\xi_{3}\right) \\
\tilde{P}(2 \Delta h)=P\left(\xi_{4}\right) \\
\tilde{P}(3 \Delta h)=P\left(\xi_{5}\right)
\end{array}\right.
$$

where $P(\xi)$ is numerical value of pressure and $\tilde{P}(\xi)$ is interpolant. The extrapolating pressure at boundary point is evaluated at $\xi=\xi_{1}$ as $\tilde{P}(-\theta)$, where $\theta$ is the fraction of cell occupied by fluids domain. Another possible scenario is that the point at $\xi=\xi_{2}$ is dropped. In such case the interpolation stencil for equation (36) and (37) should be shifted to left by one point, e.g. $\tilde{P}(0)=P\left(\xi_{3}\right)$. The extrapolating pressure is evaluated at $\xi=\xi_{1}$ as $\tilde{P}(-(\theta+\Delta h))$.

The high-order extrapolation along $\xi$ or $\eta$ direction up to second-order is employed to extrapolate flow variables from the fluid domain into the boundary. Two adjacent points in one direction associated with the boundary point are chosen to be included in the extrapolation stencil. The rule of thumb for selecting the extrapolation stencil is to avoid using flow variables in dropped points. This kind of screening criteria could ensure only irregular points can be chosen in third or high order simulation thus preventing producing non-physical solutions near solid interface boundary and improving the overall stability of simulation.

\section{Recovery process in dropped points}

It should be noted that finite difference scheme for regular or irregular points in the direction other than the prespecified direction ("dropped direction") may involve some dropped points in its stencil. As shown in Fig 5, it is not safe to abandon the dropped points in all direction, since in that case the yielding stencil for some irregular or regular points may contain a significant large interval $\theta$ compared with normal grid space $\Delta h$, which may deteriorate the high-order finite difference. Thus we should recalculate the numerical value of flow variables in these dropped points in order to make them available to use while implementing relevant numerical scheme at each time step.

A cubic interpolation is employed to interpolate the numerical values from flow variables along $\xi$ or $\eta$ direction. By the definition of dropped point, there is at least one direction in which the distance between the dropped point and some boundary point remains to be smaller than the pre-specified critical ratio. In such case the other side should be chosen for interpolation. It is also possible that in both sides the distances are smaller than the critical ratio. In such circumstance the direction with larger distance should be selected.

A general two steps algorithm for obtaining points for interpolation is depicted as follows,

1. Start at the dropped point with coordinate $\left(\xi_{i}, \eta_{i}\right)$ and set $t=0, k=0$. Assume that we move along $\xi$ direction. $t$ denotes the number of points being selected. 
2. Set $k=k+1$ and examine points at $\left(\xi_{i \pm k}, \eta_{i}\right)$. If the point at $\left(\xi_{i+k}, \eta_{i}\right)$ is not dropped point then $t=t+1$, if point $\left(\xi_{i-k}, \eta_{i}\right)$ is not dropped point then $t=t+1$. During the examining process, if $t=3$ then exit the subroutine with three point stencil, e.g. $\left(\xi_{s 1}, \xi_{s 2}, \xi_{s 3}\right)$ otherwise repeat step 2.

The interpolation value $F$ at $\left(\xi_{i}, \eta_{i}\right)$ can be calculated via evaluating the equation

$$
F(\xi)=\sum_{i=1}^{3}\left(\frac{\left(\xi-\xi_{s 1}\right)}{\left(\xi_{s i}-\xi_{s 1}\right)} \frac{\left(\xi-\xi_{s 2}\right)}{\left(\xi_{s i}-\xi_{s 2}\right)} \frac{\left(\xi-\xi_{s 3}\right)}{\left(\xi_{s i}-\xi_{s 3}\right)} F_{s i}\right.
$$

\section{Results and Discussions}

\section{A. Steady Base Flow without Roughness}

A test case for flow over a two-dimensional flat plate with Mach number 5.92 without roughness is conducted first. The numerical results are used to provide the inlet condition for region containing roughness.

The steady mean flow solutions are calculated by using a fifth-order shock fitting method discussed in section two. But in the leading edge of the flat plate, there is a singularity when high-order shock fitting method is implemented. Thus a second-order TVD shock-capturing method is employed to calculate the flow field in the tip of flat plate. The computational domain for TVD starts from a very short distance downstream of the leading edge which is divided to $100 \times 200$ grids. The ambient flow conditions are following Maslov's experiment [46] as,

$$
\begin{aligned}
& \text { - } \quad M_{\infty}=5.92, T_{\infty}=48.69 \mathrm{~K}, P_{\infty}=742.76 \mathrm{~Pa} \\
& \text { - } \quad P_{r}=0.72, R_{\infty}=\rho_{\infty} * u_{\infty} / \mu_{\infty}=1.32 \times 10^{6} / \mathrm{m}
\end{aligned}
$$

Where $M_{\infty}$ is Mach number, $T_{\infty}$ is temperature, $P_{\infty}$ is pressure, $P_{r}$ is Prantle number and $R_{\infty}$ is unit Reynolds number. The flat plate is assumed to be isothermal with fixed temperature $T_{w}=350.0 \mathrm{~K}$. The spatial discretization of TVD scheme follows Lee et al. in [47] is applied toward equation (10) and leads to semi-discrete system of ordinary differential equations, which in turn are solved by using second-order Runge-Kutta method.

To validate the numerical simulation results, we use parameters after the shock to non-dimensionalize the flow field and compare the numerical and self-similar analytical solutions from at $x=0.001 \mathrm{~m}$ in Fig. 6 . A good agreement between these two results is achieved. The minor discrepancy outside the boundary layer is caused by the shock generated by the leading edge. The two-dimensional flow field then could serve as inlet condition for the fifth-order shock fitting simulation in later zones.

The computational domain for the fifth-order shock-fitting methods starts at $x=0.003 \mathrm{~m}$ and ends at $x=1.68784 m$. In actual simulations, the computational domain is divided into 30 zones, with total of 5936 grid points in the stream wise direction, and 121 points in the wall-normal direction. The same exponential stretching function is used for coordinate transformation in the wall-normal direction in order to cluster more girds inside the boundary layer as discussed in section 2. As mentioned above, the second zone uses the results of the first zone of second-order TVD scheme as the inlet condition. The later zone used the interpolation of former zone's data as the inlet condition.

In zone 7, computational domain starts at $x=0.159 \mathrm{~m}$ and ends at $x=0.195 \mathrm{~m}$. Pressure and velocity contours for zone 8 are plotted in Fig. 8. The numerical solution is bounded by oblique shock and laminar boundary layer near the plate. Fig. 8 shows the streamwise velocity profile and temperature profiles in the wall-normal direction at the location of $x=0.1676 \mathrm{~m}$. The current numerical solutions are compared with the self-similar boundary layer solution. The velocity and temperature are normalized by corresponding freestream boundary condition, while y is nondimensionalized by $\sqrt{x \mu / \rho U}$. Fig 6-8 illustrate that the results of the current numerical simulation agree very 
well with theoretical solutions. Thus second-order TVD scheme is accurate enough to be the supporting inlet condition of fifth-order shock-fitting method.

\section{B. Steady Flow with Roughness}

A elliptic roughness shape is used in our simulation and mounted on the surface of plate at downstream $x=0.185 \mathrm{~m}$. Following Whiteheard's experiments in [43], the surface roughness is modeled as a two-dimensional bump, governed by the elliptic equation as,

$$
\frac{\left(x-x_{c}\right)^{2}}{a^{2}}+\frac{y^{2}}{b^{2}}=h^{2}
$$

The computation are performed under parameter configuration $a=2, b=1$ and $h / \delta=1 / 2$ where $\delta$ corresponds to the boundary layer thickness at $x_{c}=0.185 \mathrm{~m}$ as shown in Fig. 9. By substituting equation (40) into coordinate transformation relation (10), the analytical equation for roughness in computational domain can be written as

$$
\begin{gathered}
f(\xi, \eta)=\frac{(L \xi)^{2}}{a^{2}}+\frac{\ell H\left(\xi L+x_{\text {start }}\right)^{2}}{b^{2}}-h^{2}=0 \\
\text { with } \ell=\frac{\left(\frac{\beta+1}{\beta-1}\right)^{(1-\eta)}-1}{\left(\frac{\beta+1}{\beta-1}\right)^{(1-\eta)}+1} \beta
\end{gathered}
$$

For the sake of obtaining the boundary points on the surface roughness, a Newton iteration method is employed to solve the equation (34) with given $\xi$ or $\eta$ in range $[0,1]$. The proposed high-order cut cell method is applied to simulate the boundary layer transition with surface roughness. The overall accuracy of simulation is determined by the order of scheme in inviscid flux term. A third order converged case is obtained.

Fig. 10 shows the wall-normal velocity contours and distribution of streamlines around roughness in zone 8 . Two set of grids $240 \times 121$ and $440 \times 121$ are used to conduct the same boundary-layer transition simulation in one zone. The good agreement of these two results as shown in Fig. 10 suggests that a $240 \times 121$ resolution is accurate enough for mean flow simulation. By integrating the local velocity, the yielding streamlines distributions show that the flow is separated into two braches as approaching the roughness. The lower branch of separation flow enclosed by the roughness surface and upper branch forms a circulation. There is another circulation exist in the tail of the localized roughness with weaker strength. The streamlines also imply that the local flow around roughness don't obey the parallel assumption for linear stability calculation.

Fig. 11 shows the pressure contour in longer domain from zone 5 to zone 10. A bow shock is generated in front of the roughness via Mach wave interactions. The shock interface extends in the direction in accordance with the slope of roughness surface. Keeping tracking the flow variables in surface of roughness, the pressure reach its maximum and minimum value at the head and tail for roughness. The reason is that coming hypersonic fluids is compressed substantially by the roughness initially and then expand in the downstream. Thus a low pressure region may emerge under the shock where circulation exists.

Fig. 12,13,14 show the wall-normal velocity and density profiles in the wall-normal direction at location $x=0.188 m, x=0.200 m$ and $x=0.240 m$ for flow without and with roughness height $h / \delta=1 / 2$ respectively. The mean flow is distorted immediately after the roughness and a main steady base flow modification is formed and begins to evolutes into downstream. The amplitude of base flow modification is significant compared with the boundary layer flow initially at $x=0.188 \mathrm{~m}$. At $x=0.200 \mathrm{~m}$ the magnitude of this modification is reduced substantially but the strength of change of the density is preserved. At $x=0.240 \mathrm{~m}$ as shown in Fig. 14, the diminishing rate of strength of base flow modifications is much slower than those in previous region, which in turn imply that the oblique shock effect to the mean flow could be permanent and last in the entire simulation. It is also suggested that in current simulation region no transient growth is observed, which is consistent with Tumin's 
conclusion for two-dimensional roughness. In streamwise direction, the main roughness perturbation is moving upward constantly with some small secondary stability waves developed inside of itself.

Current computational results suggest that under the impact of roughness effect the flat-plate boundary layer is modified significantly both in the downstream and upstream region. The parallel flow assumption is violated while conducting linear stability analysis. Thus a different instability mechanism can be introduced in roughness region. But the strength of modification to mean flow with roughness height $h / \delta=1 / 2$ keeps decaying, which imply that the disturbance may not be strong enough to lead to bypass transition to turbulence directly.

\section{Unsteady Flow with Roughness}

A blow-suction slot is imposed as periodic-in-time boundary conditions for the perturbation of the mass flux on the wall. The blow-suction is located at $x=0.030 \mathrm{~m}$ and spread over several girds spaces. The perturbation is controlled by the function:

$$
\rho v=q_{0} g(l) \sum_{n=1}^{15} \sin \left(w_{n} t\right)
$$

where $q_{0}$ is an amplitude parameter and $w_{n}$ is circular frequency of this multi-frequency perturbation. $g(l)$ is nondimensional $\mathrm{x}$-direction profile function defined as

$$
g(l)=\left\{\begin{array}{l}
20.24 l^{5}-35.4375 l^{4}+15.1875 l^{3} \quad(l<1) \\
-20.24(2-l)^{5}+35.4375(2-l)^{4}-15.1875(2-l)^{3} \quad(l \geq 1)
\end{array}\right.
$$

The variable $l$ is the non-dimensional parameter associated with current coordinate of this blow-suction,

$$
l(x)=\frac{2\left(x-x_{i}\right)}{\left(x_{e}-x_{i}\right)}
$$

A fifth order shocking fitting method combined with high order upwind finite difference scheme is used for computing the unsteady Mach number 5.92 flow over flat plate with blow-suction located near the leading edge without surface roughness. At the beginning of simulation, a basic frequency $f_{1}=50 \mathrm{kHz}$ is specified and the other 14 different frequencies are integer times the basic frequency as $n=2,3,4 \ldots$ or $f=100,150,200 \ldots k H z$. This case is very similar to the simulation conducted by Wang et al. in [44].

Fig. 15 shows the pressure disturbance for the overall unsteady flow from zone 3 to zone 10. Fig. 16 shows the contours for pressure and wall-normal velocity disturbance with respect to $f=100 \mathrm{kHz}$ in zone 8 . The amplitude of disturbance is very strong initially due to the periodic injection of mass flux and then it decays fast outside the boundary layer. This damping behavior of large perturbations induced by blow-suction is very similar to the roughness effect which is considered to be caused by viscous dissipations. Due to the external unsteady disturbance, the T-S instability waves in continuous or discrete spectrum are excited inside the boundary layer via receptivity process. Fig. 17 shows the contours for pressure and wall-normal velocity disturbance with respect to $f=100 \mathrm{kHz}$ in zone 8 for flow with surface roughness. Compared with case without roughness, the behavior of the unstable waves for case with roughness is very similar in the region where roughness effect to mean flow is trivial. But as approaching the roughness, the unstable waves are shattered into several small pieces with their wave profiles distorted dramatically. In the region around the roughness, the parallel flow assumption for linear stability analysis is violated. Thus there may be no first or second mode instability behavior for T-S waves inside the boundary layer can be observed. Fig. 18 shows pressure contours for unsteady Mach 5.92 flow over a flat plate zone 9 and zone 10 with and without roughness and Fig.19 depicts the overall picture for unsteady flow with roughness from zone 3 to zone 10. The maximum pressure disturbance is reduced significantly after the roughness compared with case without roughness. Thus the local surface roughness could potentially stabilize the T-S waves in boundary layer.

Fig. 20, 21 shows the maximum magnitude of horizontal pressure disturbance along the streamwise direction with respect to different frequency, e.g. $n=1 \ldots .15$ for case with and without roughness. A Fast Fourier Transformation (FFT) technique is employed to separate all the disturbances with different frequencies. As shown in Fig. 20, the disturbances with high frequency are still stable in current region with positive growth rate. For the simulation of unsteady flow without roughness, two behaviors of evolution of T-S waves are observed. The first one is that the amplitude of T-S waves with some frequencies oscillates in the boundary layer but its variation remains within a small range, which suggests that all discrete modes are staying in upper stable region of complex plane in current 
Reynolds number. The other kind of behavior is that the amplitude is monotone increasing. As shown in Fig. 21(a), (c), compared with other frequencies, the growth rate of streamwise velocity disturbance reach the maximum at frequency $f=100 \mathrm{kHz}$. It implies that the underlying first Mack mode is unstable. For case with surface roughness as shown in Fig.21(b),(d), the amplitude of T-S waves with $f=100 \mathrm{kHz}$ decay exponentially in the region near the roughness. It might be caused by the local low speed and high viscous mean flow. In the downstream of roughness, the perturbation amplitude is reduced significantly by $70 \%$ compared with unsteady flow without roughness. For the case with roughness and $f=150 \mathrm{kHz}$, the unstable perturbation strength is much higher in the initial upstream region but decay and oscillate afterward. This kind of behavior suggests that due to the existence of surface roughness, the perturbation amplitude is being affected and dragged down, which may lead to delay the possible bypass transition in further downstream.

A linear stability analysis is applied to analyze the flow field with surface roughness. The small perturbation in the flow field can be written in the form of normal mode (T-S waves):

$$
\left\{\begin{array}{l}
u^{\prime} \\
v^{\prime} \\
w^{\prime} \\
p^{\prime} \\
\theta^{\prime}
\end{array}\right\}=\left\{\begin{array}{l}
\tilde{u}(y) \\
\tilde{v}(y) \\
\tilde{w}(y) \\
\tilde{p}(y) \\
\tilde{\theta}(y)
\end{array}\right\} e^{i\left(\int \alpha d x+\beta d z-\omega t\right)}
$$

Where $\theta$ is the perturbation of temperature, $\omega$ is circular frequency.

After substituting Equation (45), the equation (1) can be linearized by omitting small high-order terms. A mutidomain spectral collocation method is used first to discretizate the linearized version of equation (1) and obtain the global spectrum of T-S waves following Malik's paper [48]. The coarse eigenvalues are then purified by using a second-order finite difference method. More details about these methods can be found in [48].

The non-dimensionalized frequency used for LST analysis is obtained by,

$$
F=\frac{2 \pi f v}{U_{\infty}^{2}}
$$

Where $F$ is nondimensionlized frequency with respect to viscosity scale, $v$ is kinematic viscous coefficient. In present simulation, $v=6.05 \times 10^{-5} \mathrm{~m}^{2} / \mathrm{s}, F=5.30 \times 10^{-5}$ with respect to $f=100 \mathrm{kHz}$ and $U_{\infty}=827.29 \mathrm{~m} / \mathrm{s}$.

Fig. 22 shows the discrete and continuous spectrum of $\alpha$ for flow in region with different distance to roughness. Fig. 23 depicts the streamwise velocity and pressure disturbance profile for corresponding discrete eigenmode. The disturbance amplitude are nondimensionlized by its corresponding value on the surface of flat plate, e.g. $u^{\prime}(y)=\tilde{u}(y) / \tilde{u}(0)$.In the upstream of roughness $x=0.1620 \mathrm{~m}$ where the roughness effect is trivial, the Mack mode $\mathrm{S}$ and mode $\mathrm{F}$ can be indentified clearly in Fig. 23. As the flow intersection for LST analysis moving gradually to downstream, the spectrum of normal eigenmode varies significantly. By tracking the location of Mode $\mathrm{S}$ and Mode $\mathrm{F}$ in region close to roughness, at $x=0.1740 \mathrm{~m}$ Mode $\mathrm{F}$ disappear and Mode $\mathrm{S}$ become more unstable compared with case without roughness. By analyzing the unstable eigenmode profile in roughness region as shown in Fig. 23 (c), (d), a very different eigenmode profile is observed. The streamwise velocity disturbance profile of this unstable eigenmode is less peaked, but with more wave crests and troughs inside the boundary layer than Mode $\mathrm{S}$. The pressure disturbance profile exhibits negative value in curtain range away from the wall. Those extraordinary characters suggest that this unstable discrete eigenmode is not typical Mode $\mathrm{S}$ in boundary layer flow, thus we refer it to as Mode $\mathrm{S}^{*}$. In the region immediate after the roughness, the Mode $\mathrm{S}$ and Mode $\mathrm{F}$ can not be identified and the continuous entropy and vorticity spectrum is discretized up to location at $x=0.1980 \mathrm{~m}$ as shown in Fig.22 (c). But in the downstream of roughness starting from $x=0.1920 \mathrm{~m}$, Mode $\mathrm{S}$ and Mode $\mathrm{F}$ can be identified again by examining their perturbation profiles in Fig. 23. There will be an oscillation in Mode S and Mode F's velocity perturbation profile around $Y^{*}=50$, which imply that the oblique shock generated by roughness would affect the eigenmode profile.

Fig. 24 and 25 shows variation of wave speed $a$ and growth rate $\alpha_{i}$ of Mode $\mathrm{S}$ and Mode F vs x(m) for unsteady flow without and with roughness via LST analysis. The wave speed is defined as 


$$
a=\frac{F \mathrm{Re}}{\alpha_{r}}
$$

where $F$ is nondimensionlized frequency and Re is local Reynolds number.

As shown in Fig.24(a) and 25(a), compared with unsteady flow without roughness, the wave speed variation for case with roughness in region $x=0.195 \sim 0.253 \mathrm{~m}$ does not change much. There are two discrepancies between the two shown in the Fig. 24 and 25. The first discrepancy is in region where Mode F moves into the continuous entropy and vorticity spectrum. Inside this region it is difficult to track the accurate location of Mode F thus this discrepancy in Mode F branch can be ignored safely. The second discrepancy is that the syncolization point of Mode F and Mode $\mathrm{S}$ for unsteady flow with roughness shift to left. Thus due to the roughness effect, the Mode S may turn into more unstable Second Mode with maximum growth rate $\alpha_{i}$ earlier via eigenmode interactions.

In Fig. 24(b) and 25(b), it is worthy noting that the growth rate $\alpha_{i}$ of Mode $\mathrm{S}^{*}$ for case with roughness is exceptionally large. This unstable behavior branches out from our direct simulation result. As shown in Fig. 21(a), the amplitude of pressure disturbance is Mode $S^{*}$ region is reduced substantially. One explanation for this result is that due to the existence of circulation inside the boundary layer as shown in Fig. 10, the parallel flow assumption is partially violated, which is critical for LST analysis. Thus in Mode $S^{*}$ region the LST result may not be accurate and can not be comparable with DNS result as enclosed in dash lines in Fig. 25(b).

Fig 21 and 25 show that our DNS result agrees well with the LST analysis in region after the roughness where parallel flow assumption is hold. Starting from location $x=0.195 m$, the Mode $\mathrm{S}$ is stable initially $\left(\alpha_{i}>0\right)$, fall into unstable region at range $x=0.195 \sim 0.198 m$ ( $\alpha_{i}$ turn into negative quickly and reach a local minimum point ), and then rebound to neutral state at range $x=0.198 \sim 0.206 m$ ( $\alpha_{i}$ approaches zero point). The trajectory of Mode $\mathrm{S}$ movement exactly matches the behavior of pressure disturbances with $f=100 \mathrm{kHz}$ as shown in Fig. 21(b). At range $x=0.220 \sim 0.250 m$, LST analysis in Fig 25(b) shows that the Mode S could be considered to be neutrally stable, which is consistent with our numerical simulation. Although the DNS is still running to further downstream, by far the LST analysis has already partially validated our DNS result.

\section{Conclusions}

For the sake of overcoming the difficulties of grids generation in arbitrary domain, in this paper we develop a high-order cut-cell method for computational domain where grids are easy to generate. A very high-order nonuniform finite difference method is employed to discretize the irregular points near the cut cells, and a prespecified critical ratio is set up to prevent the potential numerical instability in simulation. The new high-order cut-cell method is successfully applied into computing Mach number 5.92 flow over flat plate with surface roughness height $\mathrm{h}=\frac{1}{2} \delta$ (small roughness). The unsteady receptivity process induced by the interaction of blow-suction and flat plate with roughness is also numerically investigated and analyzed by linear stability theory (LST).

For the steady flow, due to the existence of surface roughness the hypersonic flow is compressed and a weak oblique shock is generated in front the roughness and then an expansion waves is followed. There is a significant change of mean flow profiles inside the boundary layer with flow separation before and after the roughness element. But the modification to basic flow keeps decaying outside the boundary layer. For unsteady flow, the simulation results show that the amplitude of most unstable perturbation is reduced significantly by $70 \%$ after the roughness element compared with flow without roughness. After applying LST analysis into flow intersections in roughness downstream, the discrete Mode S and Mode F could be identified clearly with small oscillation in their perturbation profiles. With the increasing of Reynolds number, the trajectory of Mode S and Mode F eigenvalues $\alpha$ and negative peak value of Mode $S \alpha_{i \max }$ do not change much in complex plane compared with those for flow without roughness, thus our LST analysis validates our DNS result partially. The combination of LST analysis and DNS result also suggests that the existence of small surface roughness $\left(\mathrm{h}=\frac{1}{2} \delta\right)$ can delay the transition in hypersonic boundary layer. 


\section{Acknowledgments}

This work was sponsored by the Air Force Office of Scientific Research, USAF, under AFOSR Grant \#FA955007-1-0414, monitored by Dr. John Schmisseur. The views and conclusions contained herein are those of the author and should not be interpreted as necessarily representing the official policies or endorsements either expressed or implied, of the Air Force Office of Scientific Research or the U.S. Government.

\section{References}

1. E. Piot, G. Casalis and M. Terracol, Direct numerical solution of the crossflow instabilities Induce by a Periodic Roughness Array on a swept cylinder: receptivity and stability investigations, $37^{\text {th }}$ AIAA Fluid Dynamics Conference and Exhibit, Miami, FL, June 25-28, 2007 No. 1, 1999, pp. 134-150

2. S. Berry and T. Horvath, Discrete roughness transition for hypersonic flight vehicles , $45^{\text {th }}$ AIAA Aerospace Sciences Meeting and Exhibit, Reno, Nevada, Jan. 8-11, 2007

3. Malik, M.R., Spall, R. E., and Chang, C. L., Effect of nose bluntness on boundary layer stability and rransition. 1990. AIAA Paper 90-0112.

4. William S.Saric, Helen L. Reed, and Edward J.Kerschen, Boundary-Layer receptivity to freestream disturbances, Annu. Rev. Fluid Mech, 2002.34:291-319

5. T. Y. Hou, Z. Li, S. Osher, H. Zhao A hybrid method for moving interface problems with application to the Hele-Shaw flow. J. of Comp Physics.134:236-252.

6. K. Ito, Z. Li Solving a nonlinear problem in magneto-rheological fluids using the immersed interface method. 19:253-266.

7. Sheng $\mathrm{Xu}, \mathrm{Z}$. Jane Wang, An immersed interface method for simulating the interaction of a fluid with moving boundaries, J. of Comp Physics. 216: 454-493

8. D.V.Le, B.C.Khoo, J. Peraire An immersed interface method for viscous incompressible flows involving rigid and flexible boundaries, J. of Comp Physics.220:109-138

9. M. N. Linnic, F. F. Hermann A high-order immersed interface method for simulating unsteady incompressible flows on irregular domains. Journal of Computational Physics. 204:157-192.

10. B. Lombard, J. Piraux Numerical treatment of two-dimensional interfaces for acoustic and elastic waves. Journal of Computational Physics. 195:90-116.

11. Xiaolin Zhong, A new high-order immersed interface method for solving elliptic equations with imbedded interface of discontinuity, Journal of Computational Physics, 225:1066-109

12. M. Kang, R. P. Feskiw, X. D. Liu A boundary condition capturing method for multiphase incompressible flow. J. of Scientific Computing. 15:323-360.

13. Wlezien RW, Parekh DE, Island TC. 1990. Measurement of acoustic receptivity at leading edges and porous strips. Appl. Mech. Rev. 43 (Pt. 2): S167-74

14. Saric WS, Hoos JA, Radeztsky RH Jr. 1991. Boundary-layer receptivity of sound with roughness. See Reda et al. 1991, pp. 69-76

15. J.W.Purvis and J.E.Burkhalter, Prediction of critical Mach number for store configurations AIAA J. 17, 1979

16. D.K.Clarke, M.D.Salas, Euler calculations for multielement airfoils using Cartesian grids AIAA J.24

17. M.J.Berger and R.J.Leveque, Stable Boundary Condition for Cartesian Grid Calculations, Computing System, 1990

18. Hans Johansen, Phillip Colella, A Cartesian Grid Embedded Boundary Method for poisson's equation, J. Comput. Phys, 1998

19. Pember, Bell, Colella et al., An Adaptive Cartesian Grid Method for Unsteady Compressible Flow inirregular Regions, J. Comput. Phys, 2006

20. Colella et al., A node-centered local refinement algorithm for Poisson's equation in complex geometries, J. Comput. Phys, 2004

21. James J. Quirk, An alternative to unstructured girds for computing gas dynamic flows around arbitrarily complex bodies, Comp. Fluids, 1994

22. T. Ye, R. Mittal, H. S. Udaykumar and W. Shyy, An Accurate Cartesian Grid Method for ViscousIncompressible Flows with Complex Immersed Boundaries, Journal of Computational Physics, Volume156, Issue 2, 10 December 1999, Pages 209-240 
23. Hans Forrer and Rolf Jeltschy , A Higher-Order Boundary Treatment for Cartesian-Grid Methods , J.Comput. Phys, 1997

24. M,Popescu et al., A Finite Volume-Based High Order Cartesian Cut-cell Method for Computational Aeroacoustics, AIAA Paper, 2005

25. Michael Barad, Phillip Colella, A fourth-order accurate local refinement method for poisson's equation, J. Comput. Phys, 2005

26. Gibou, Fedkiw, A fourth order accurate discretization for laplace and heat equations on arbitrary domain, J. Comput. Phys, 2004

27. DD.Zeeuw and KG.Powell, An Adaptively Refined Cartesian Mesh Solver for the Euler Equations, J. Comput. Phys, 1993

28. Guang-Shan Jiang, Efficient Implementation of Weighted ENO Schemes, J. Comput. Phys 126,1996

29. J. H. Williamson, Low-storage Runge-Kutta schemes, J. Comput. Phys. 35, 48 (1980).

30. $\quad$ Frank M. White. Viscous Fluid Flow, Third Edition, 2006

31. Zhong, X., and Ma, Y., Boundary-layer receptivity of Mach 7.99 Flow over a blunt cone to free-stream acoustic waves. Journal of Fluid Mechanics, 2005. 556: p. 55-103.

32. H. S. Udaykumar, R. Mittal, P. Rampunggoon, A. Khanna A sharp interface Cartesian grid method for simulating flows with complex moving boundaries. J. Comput. Phys. 174:345-80.

33. H. S. Udaykumar, R. Mittal, W. Shyy Computation of solid-liquid phase fronts in the sharp interface limit on fixed grids. J. Comput. Phys. 153:534-74.

34. H. S. Udaykumar, W. Shyy, M. M. Rao Elafint: A mixed Eulerian-Lagrangian method for fluid flows with complex and moving boundaries. Int. J. Numer. Methods Fluids 22:691-705

35. C. Peskin The immersed boundary method. Acta Numerica. 479-517.

36. Fedkiw, R.P., Aslam, T., Merriman, B and Osher . A non-oscillatory Eulerian approach to interface in multimaterial flows(the Ghost Fluid Method) J. of Comp. Physics , 152:457-492.

37. L. Lee, R. LeVeque An immersed interface method for incompressible Navier-Stokes equations. SIAM J. Sci. Computing, 25:832-856.

38. R. LeVeque, Z. Li The immersed interface method for elliptic equations with discontinuous coefficients and singular sources. SIAM J. Numer. Anal.31:100125.

39. A. Wiegmann, K. P. Bube The immersed interface method for nonlinear differential equations with discontinuous coefficients and singular sources. SIAM J. Numer. Anal. 35:177-200.

40. A. Wiegmann, K. P. Bube The Explicit-jump immersed interface method: finit difference methods for PDEs with piecewise smooth solutions. SIAM J. Numer. Anal. 37:827-862.

41. X.Zhong, High-order finite-difference schemes for numerical simulation of hypersonic boundary- layer transtion, J. Comput. Phys 144,1998

42. J. H. Williamson, Low-storage Runge-Kutta schemes, J. Comput. Phys. 35, 48 (1980).

43. A.H. Whitehead, Jr. Flowfield and drag characteristics of several boundary-layer tripping elements in hypersonic flow. Techinical Note TND-5454, NASA October 1969

44. A. Tumin, X. Wang, and X. Zhong, Direct numerical simulation and the theory of receptivity in a hypersonic boundary layer, Physics of Fluids, 19, 014101, 2007

45. Le Duan, A High Order Cartesian Girds Method for Numerical Simulation of Hypersonic Boundary Layer Stability With Finite Surface Roughness, AIAA-2008-3732, 38th Fluid Dynamics Conference and Exhibit, Seattle, Washington, June23-26, 2008

46. H.C Yee, R.F Warming, A Harten, Implicit total variation diminishing (TVD) schemes for steady-state calculations, J. Comput. Phys, Volume 57, Issue 3, February 1985, Pages 327-360

47. B. A. Maslov, A. N. Shiplyuk, A. Sidorenko, and D. Arnal. Leading-edge receptivity of a hypersonic boundary layer on a flat plate. Jounral of Fluid Mechanics, Vol.426, pp.73-94, 2001.

48. M. R. MALIK, Numerical Methods for Hypersonic, Boundary Layer Stability, J. Comput. Phys 86, $376-413$ (1990) 


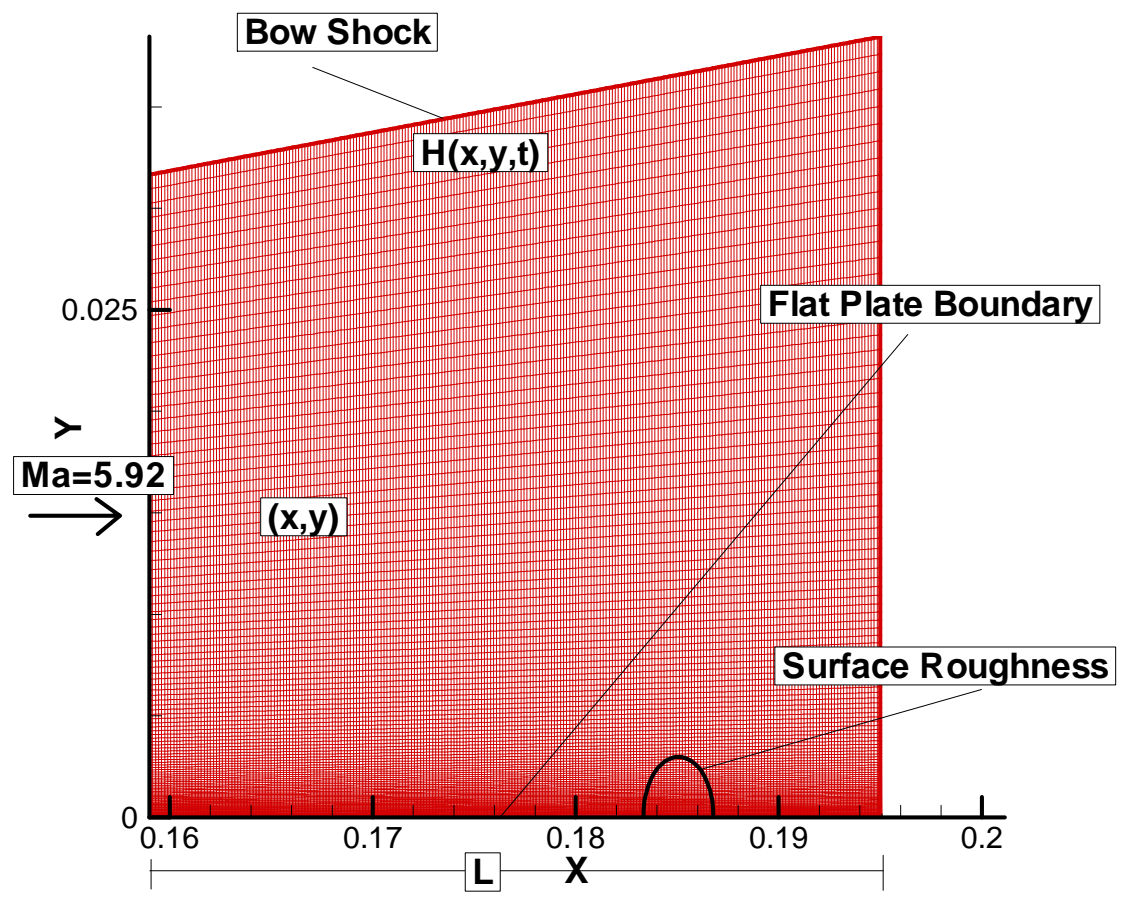

Figure 1. Body-fitted grids for flow over flat plate with finite surface roughness. 


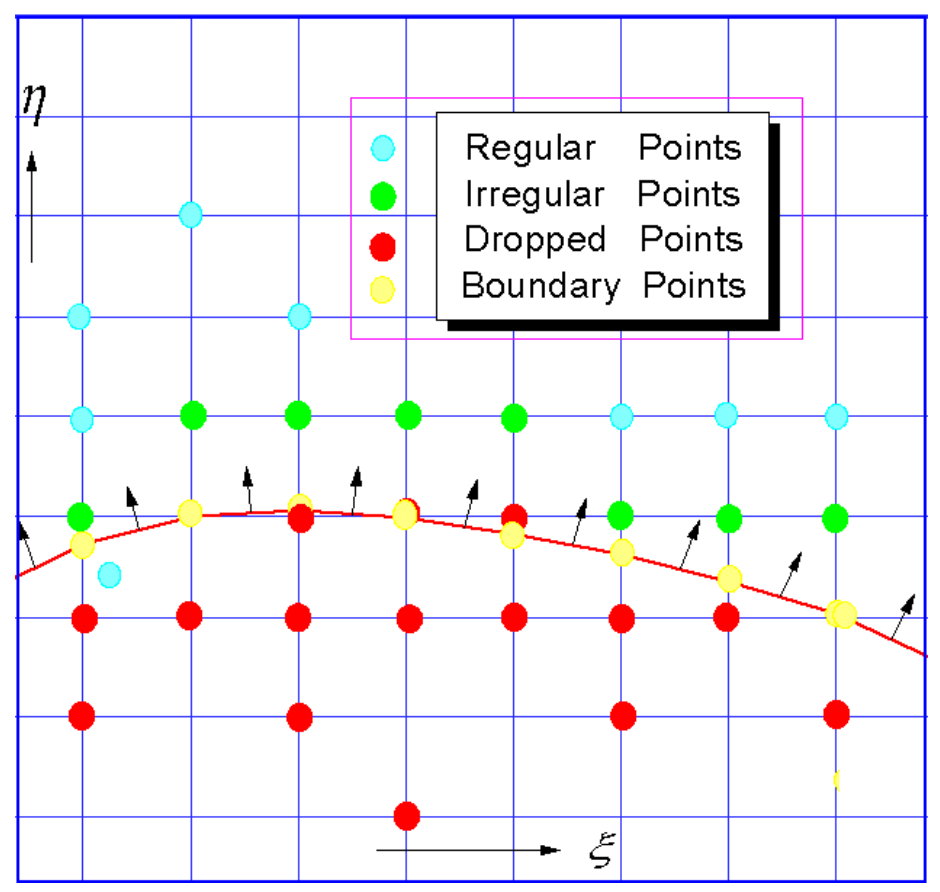

Figure 2. Definition of grids for high-order cut cell method. Four kinds of grids are defined. The intersections of roughness surface grids lines are defined as boundary points and marked as yellow dot. The grids points adjacent to a boundary point with a distance smaller than a pre-specified critical ratio in wall-normal or streamwise direction, they are defined as dropped points and marked as red dots. The irregular points are defined as whose stencil involving boundary point and marked as green dots. The rest points are defined as regular points and marked as blue dots.

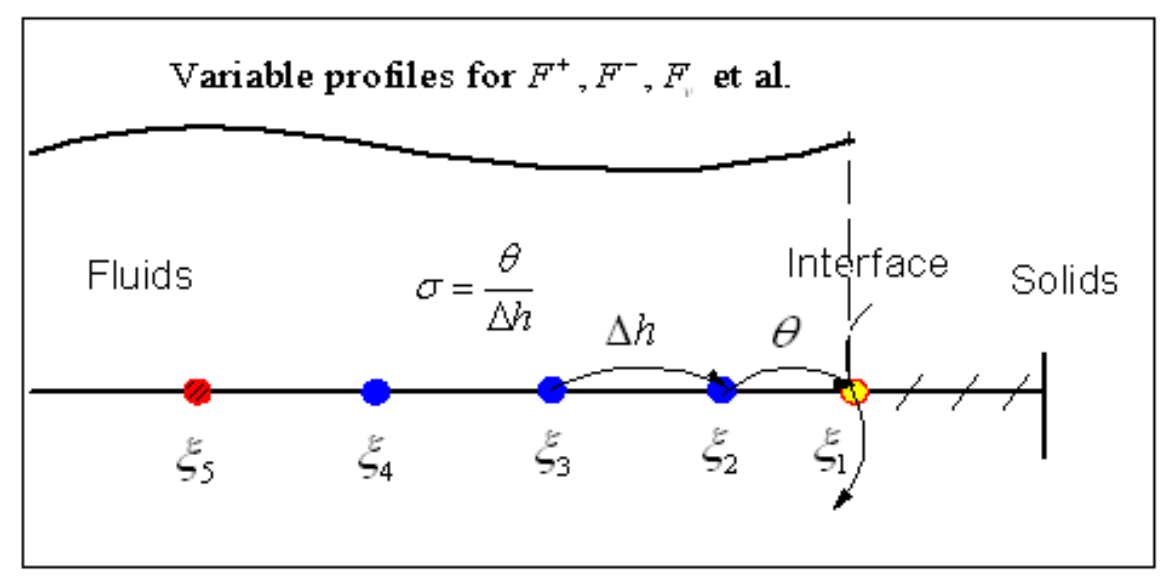

Figure 3. A fourth order stencil for discretizating viscous terms $F_{v, i}$ for $i=2,3,4$. $\theta$ is non-uniform girds space after removing the dropped points, $\Delta$ h is normal girds space.

American Institute of Aeronautics and Astronautics 


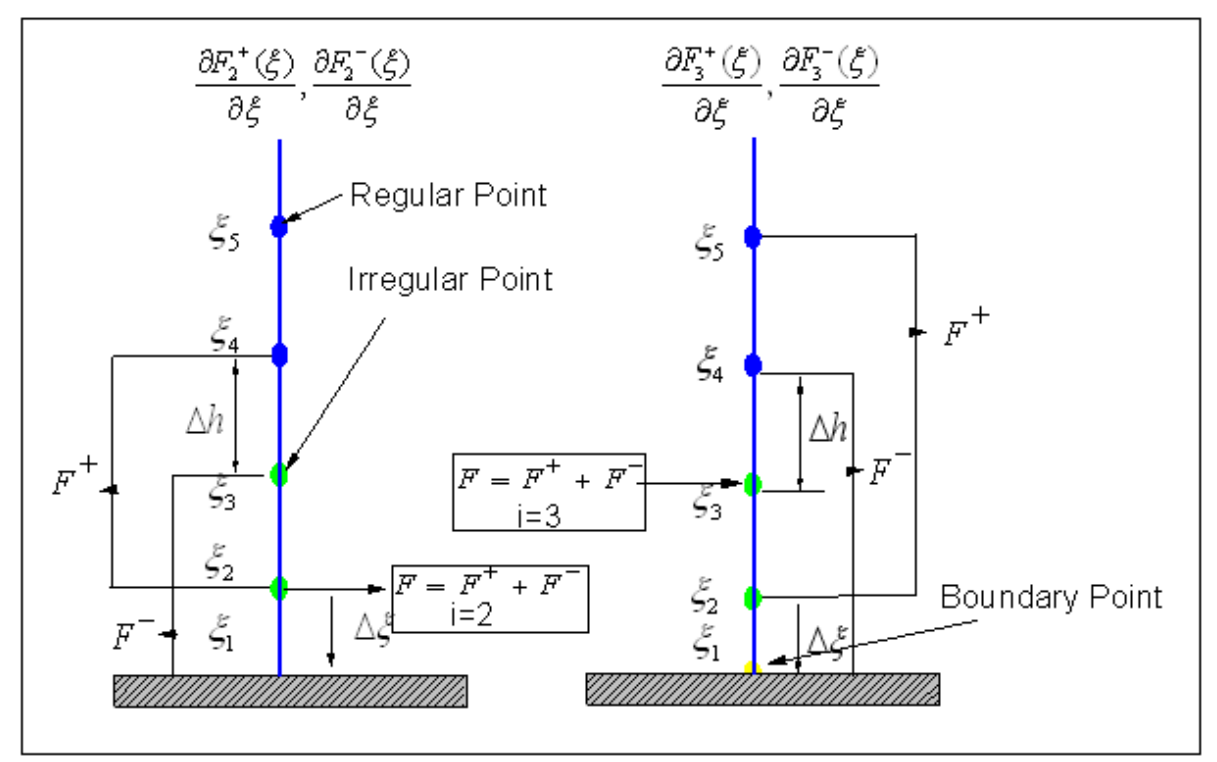

Figure 4. A stalbe third order scheme for boundary closure. For irregular point indexed by $i=3$, the stencil of upwind scheme is point set $\left(\xi_{2}, \xi_{3}, \xi_{4}, \xi_{5}\right)$ for positive flux $F^{\prime+},\left(\xi_{1}, \xi_{2}, \xi_{3}, \xi_{4}\right)$ for negative flux $F^{{ }^{-}}$. For irregular point indexed by $i=2$, the stencil of upwind scheme is $\left(\xi_{2}, \xi_{3}, \xi_{4}\right)$ for positive flux $F^{{ }^{+}},\left(\xi_{1}, \xi_{2}, \xi_{3}\right)$ for negative flux $F^{1^{-}}$.

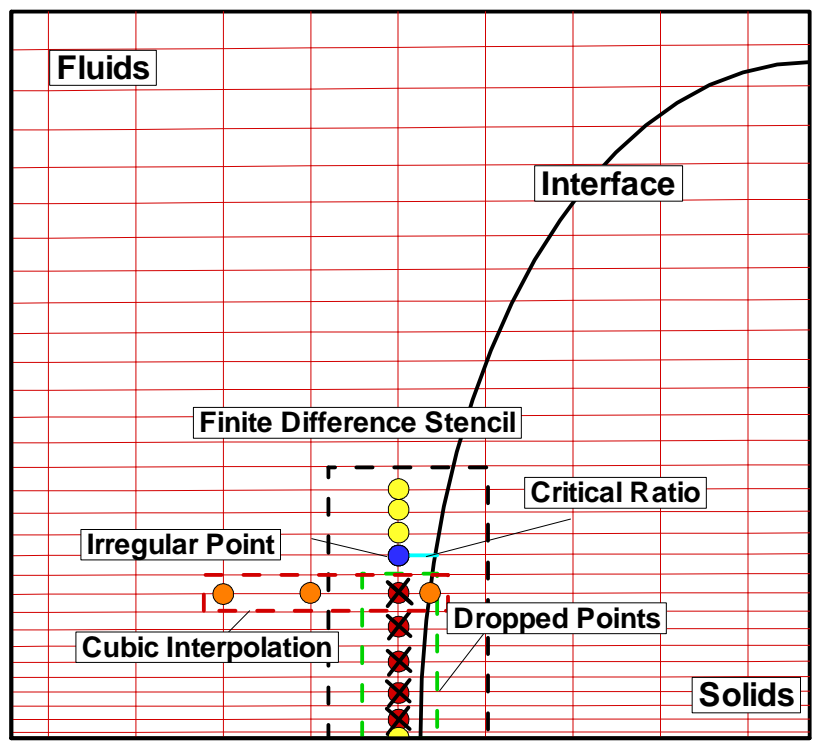

Figure 5. A special case for irregular point stencil which may contain a significant large interval compared with normal grid space if ignoring dropped points in all direction. In such case the difference stencil is consisted of yellow points, which may deteriorate the high-order finite difference. An effective way of resolving this problem is utilize a cubic interpolation in $\xi$ direction to recalculate the values in dropped points. Then the dropped point can be included into the difference stencil. 


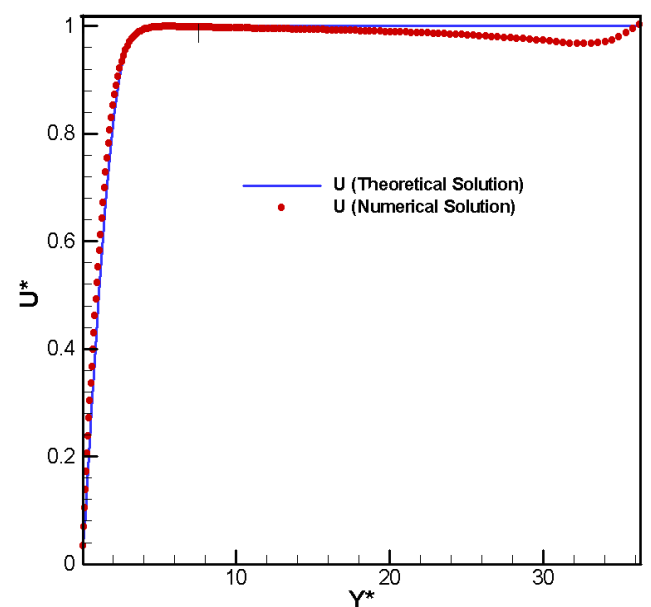

(a)

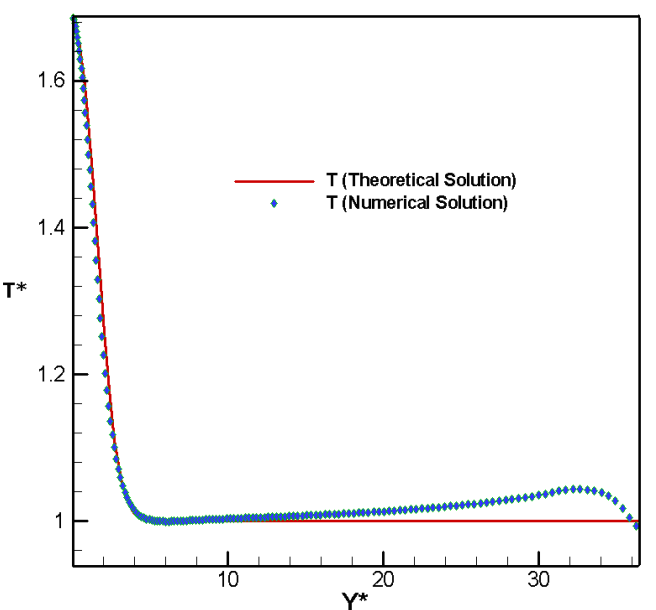

(b)

Figure 6. Along a wall-normal grid line which is originated from a point located at $x=0.001 \mathrm{~m}$ on the plate surface. (a), temperature (b), velocity in y direction profile.

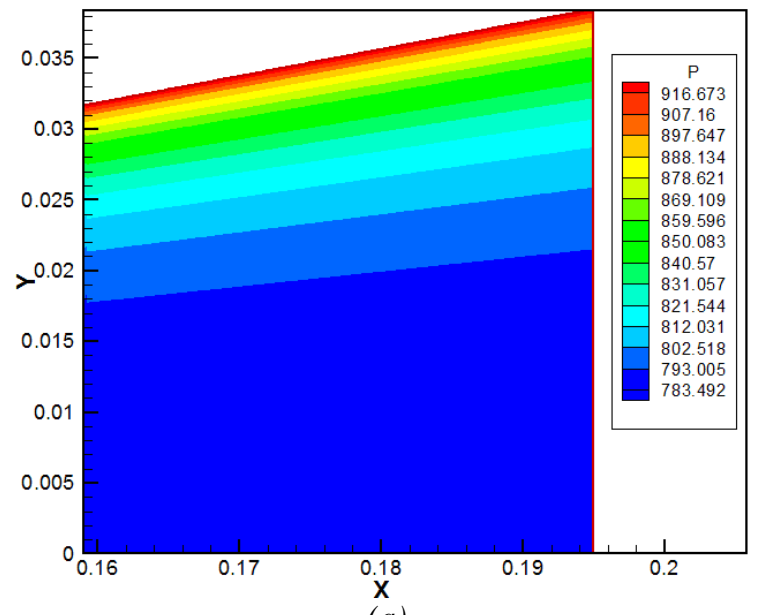

(a)

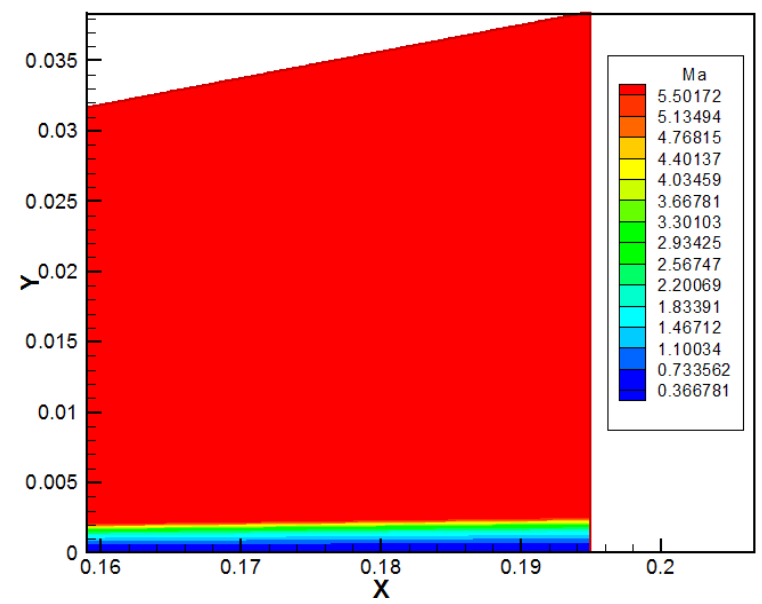

(b)

Figure 7. Contours for steady Mach 5.92 flow over a flat plate zone 8 without the roughness (a), pressures (b), velocity.

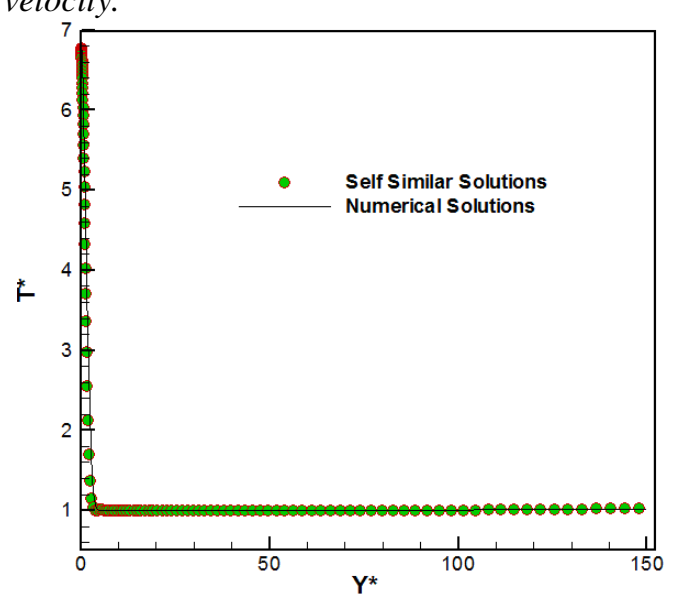

(a)

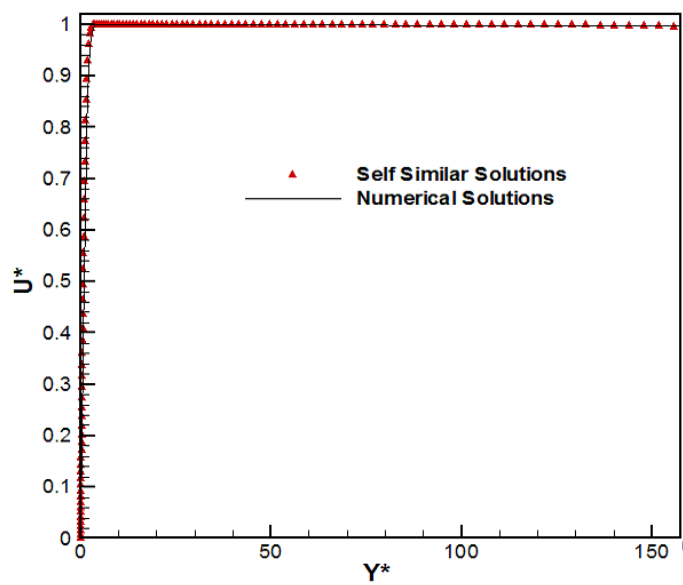

(b)

Figure 8. Along a wall-normal grid line which is originated from a point located at $x=0.1676 \mathrm{~m}$ on the plate surface. (a), temperature (b), velocity in y direction profile. 


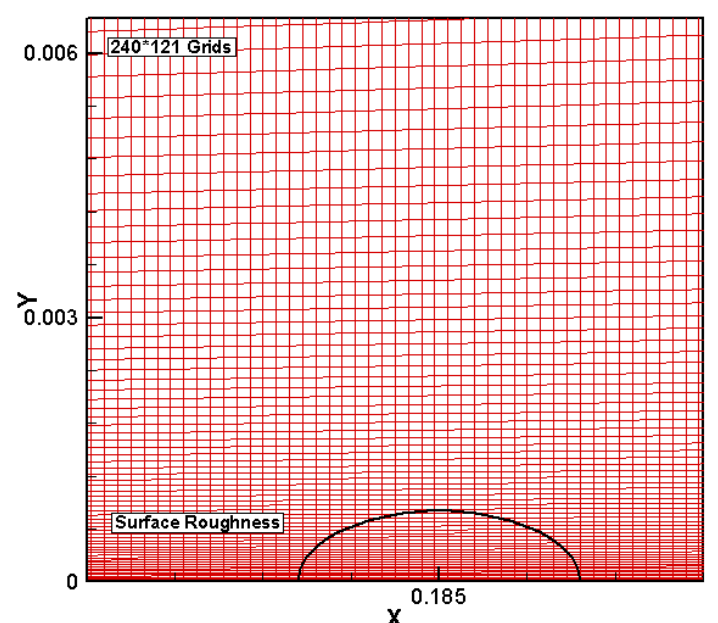

(a)

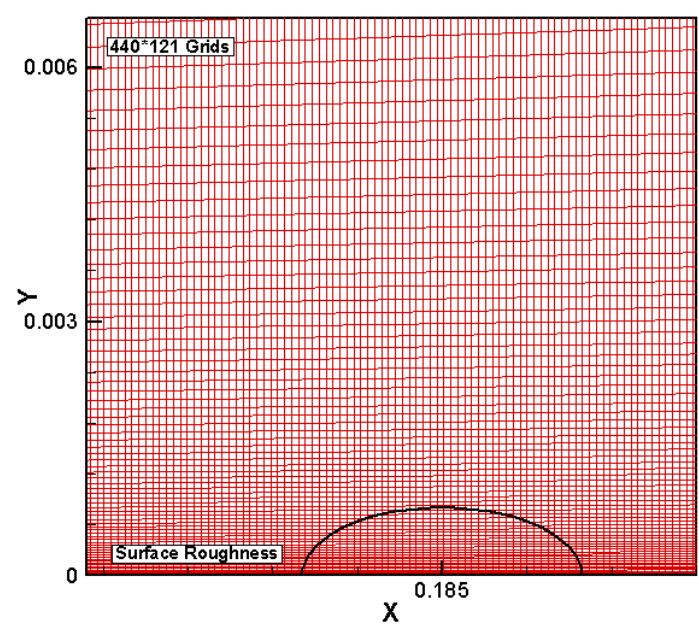

(b)

Figure 9. Grids distribution and roughness shape for (a), $240 \times 121$ case, (b), $440 \times 121$ case

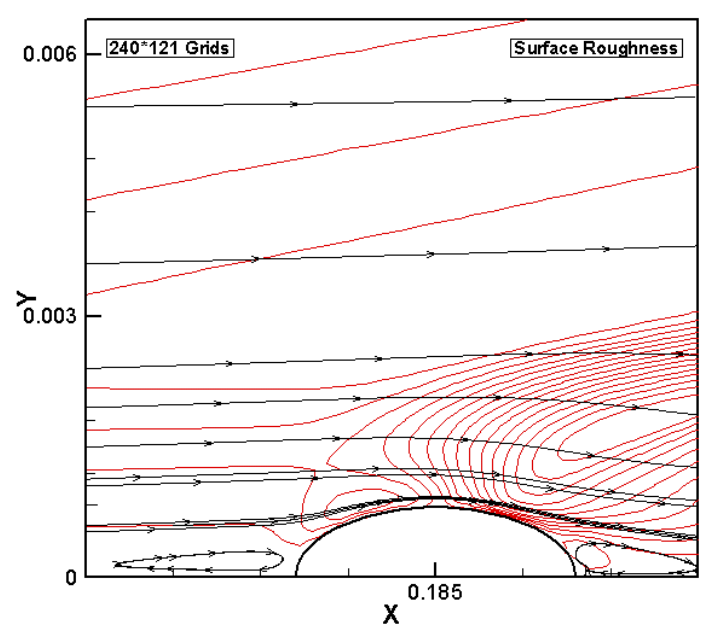

(a)

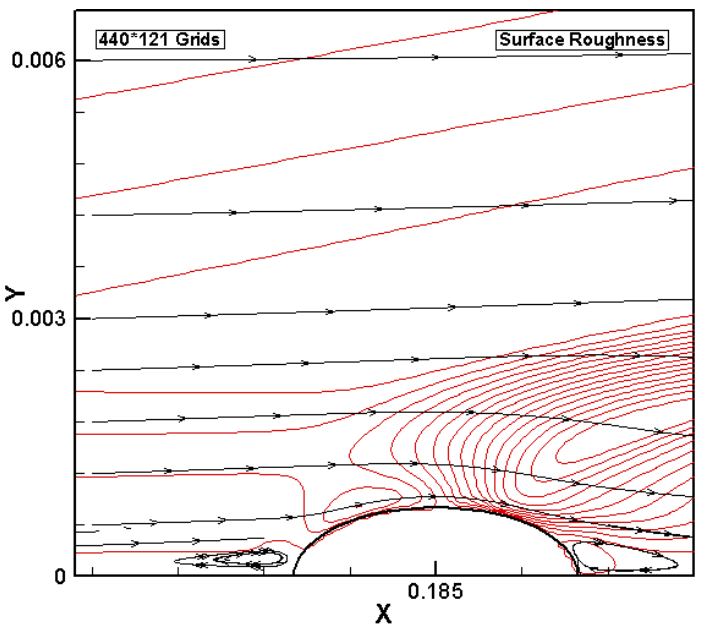

(b)

Figure 10. Contour of wall-normal velocity and distribution of streamlines for (a), $240 \times 121(b), 440 \times 121$ case with surface roughness $h / \delta=1 / 2$ at zone 8 .

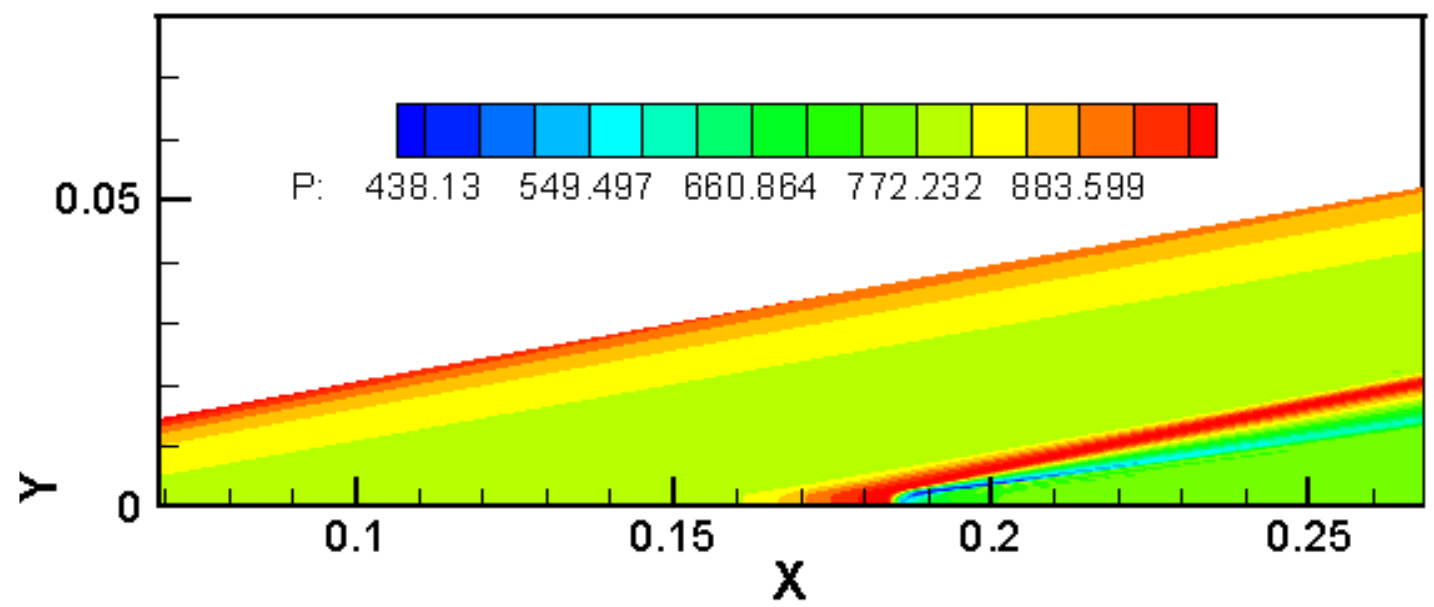

Figure 11. Pressure contours for steady Mach 5.92 flow over a flat-plate from zone 5 to zone 10 with surface roughness $h / \delta=1 / 2$. 


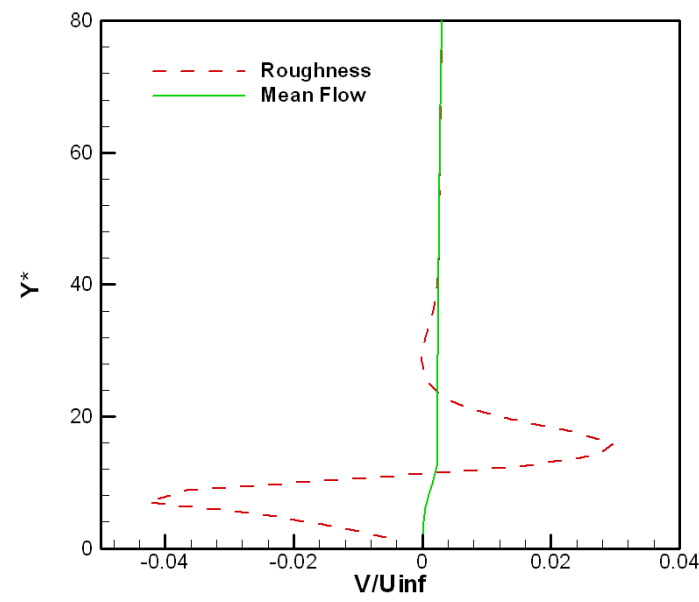

(a)

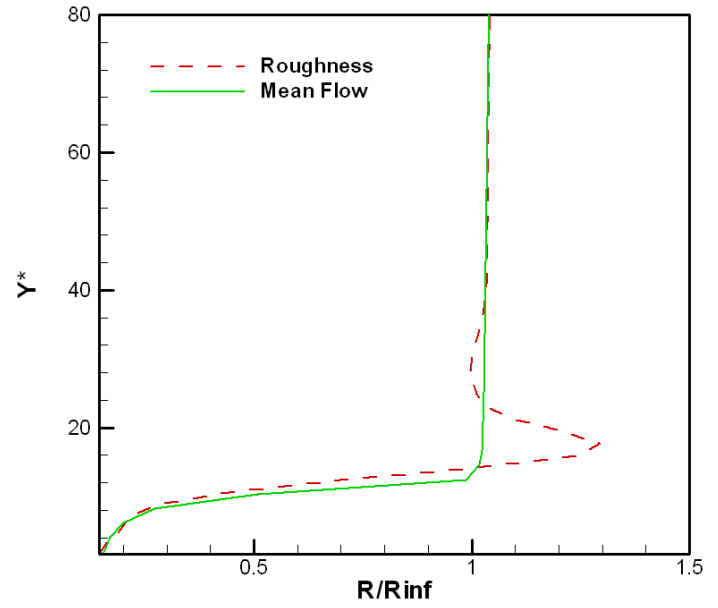

(b)

Figure 12. Along a wall-normal grid line at $x=0.188 m$ on the plate surface $(a)$, horizontal velocity (b), density profile for flow with and without roughness.
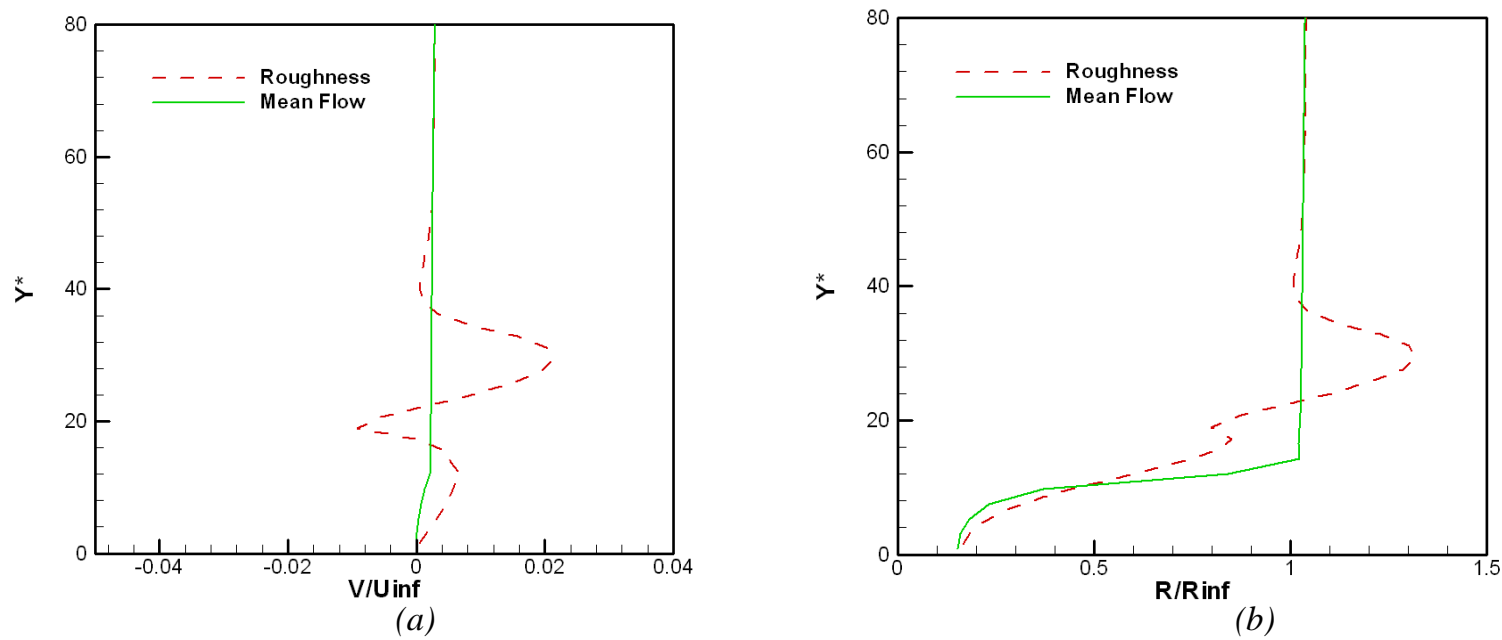

Figure 13. Along a wall-normal grid line at $x=0.200 \mathrm{~m}$ on the plate surface (a) spanwise velocity (b), density profile for flow with and without roughness.
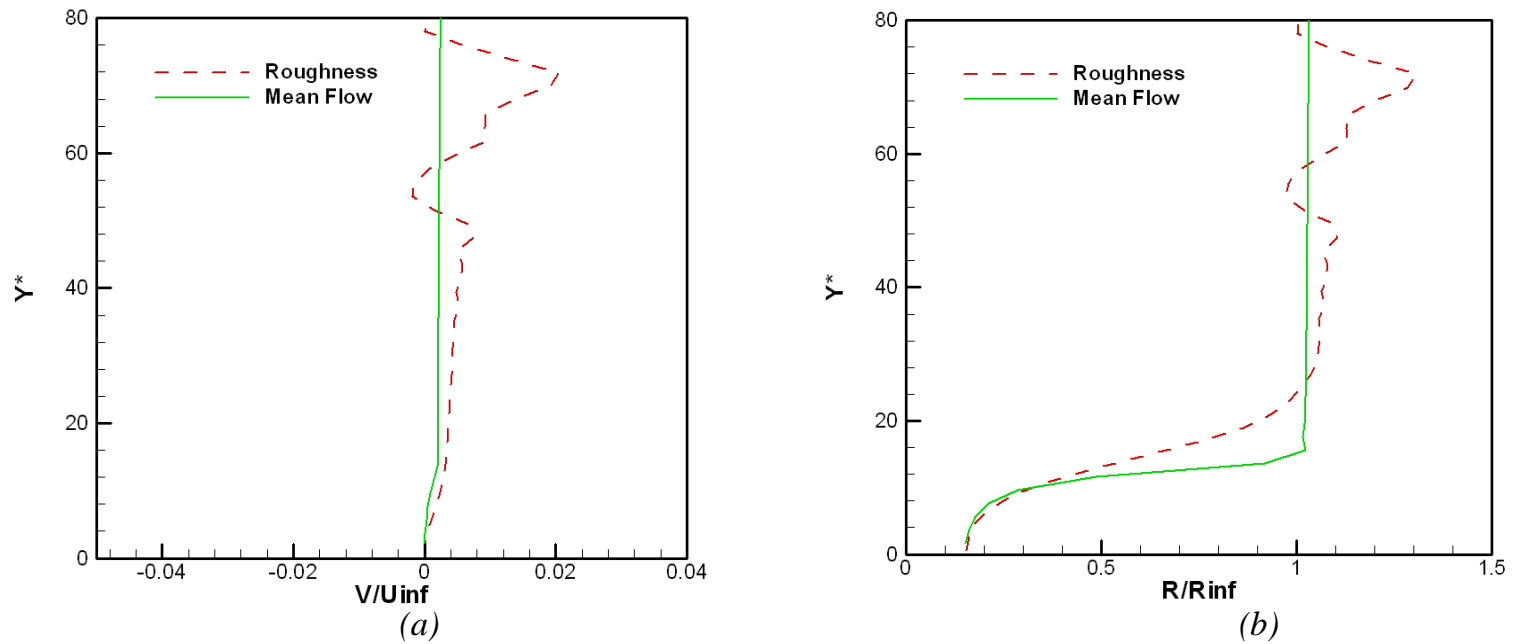

Figure 14. Along a wall-normal grid line at $x=0.240 \mathrm{~m}$ on the plate surface (a), horizontal velocity (b), density profile for flow with and without roughness.

American Institute of Aeronautics and Astronautics 


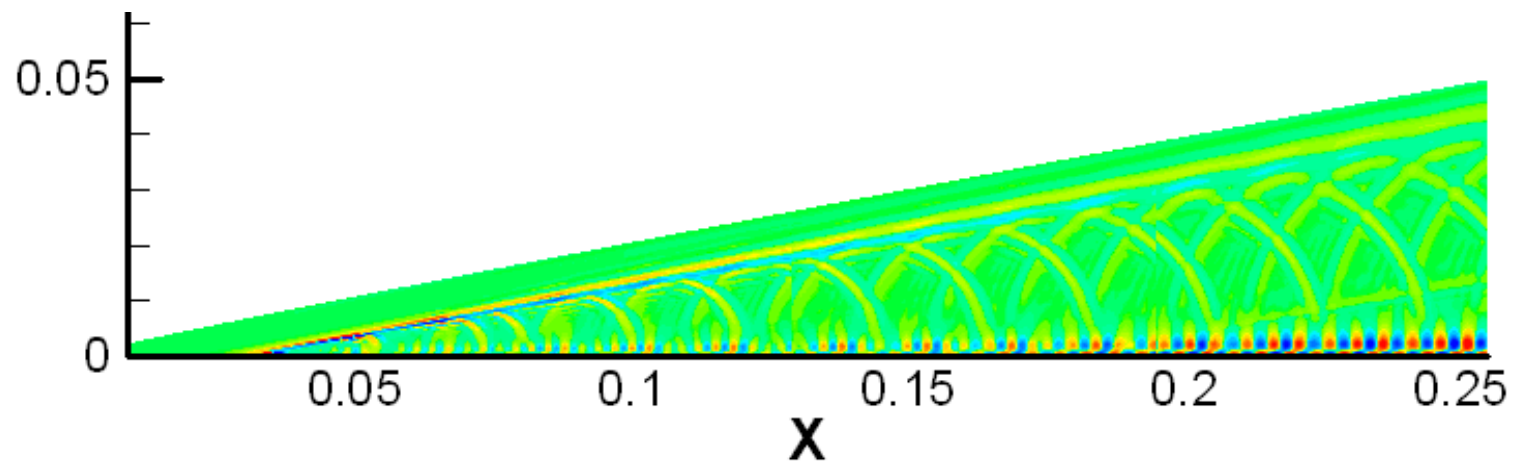

Figure 15. Pressure disturbance contour for unsteady flow field with external disturbance from zone 3 to zone 10.

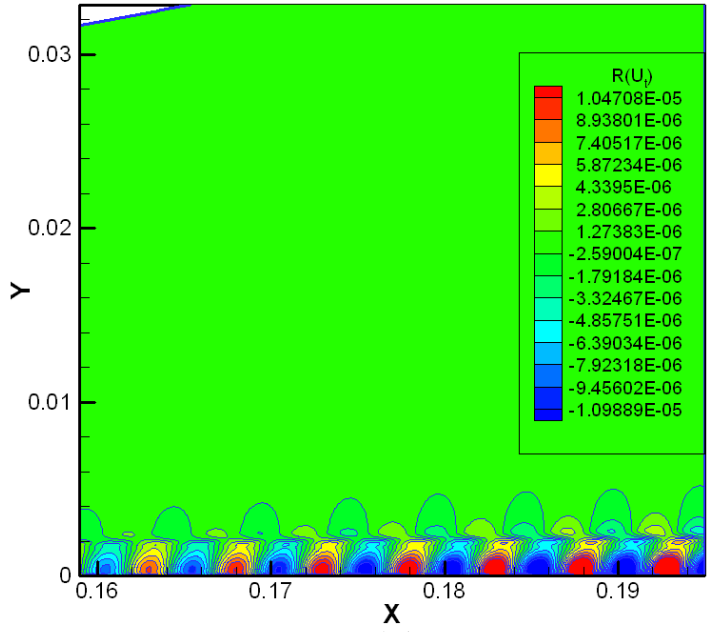

(a)

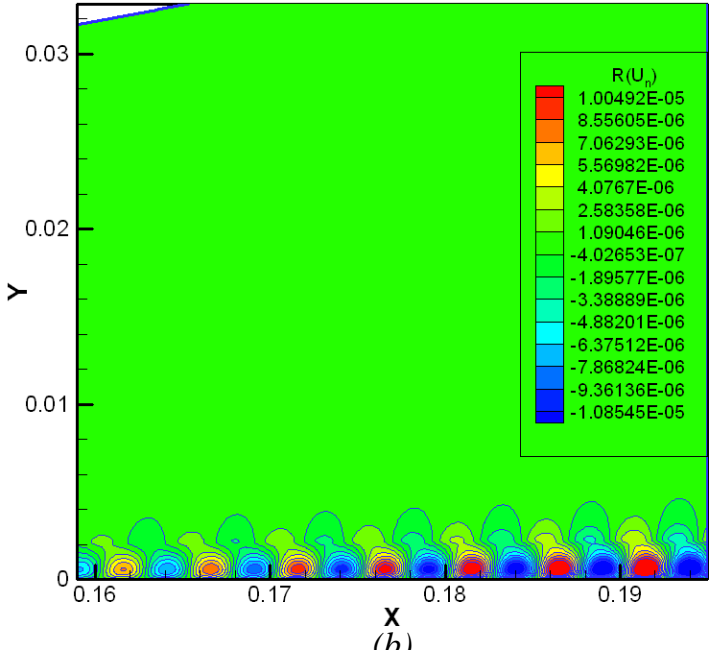

(b)

Figure 16. Contours for unsteady Mach 5.92 flow over a flat plate zone 8 without the roughness (a), pressures (b), wall-normal velocity.
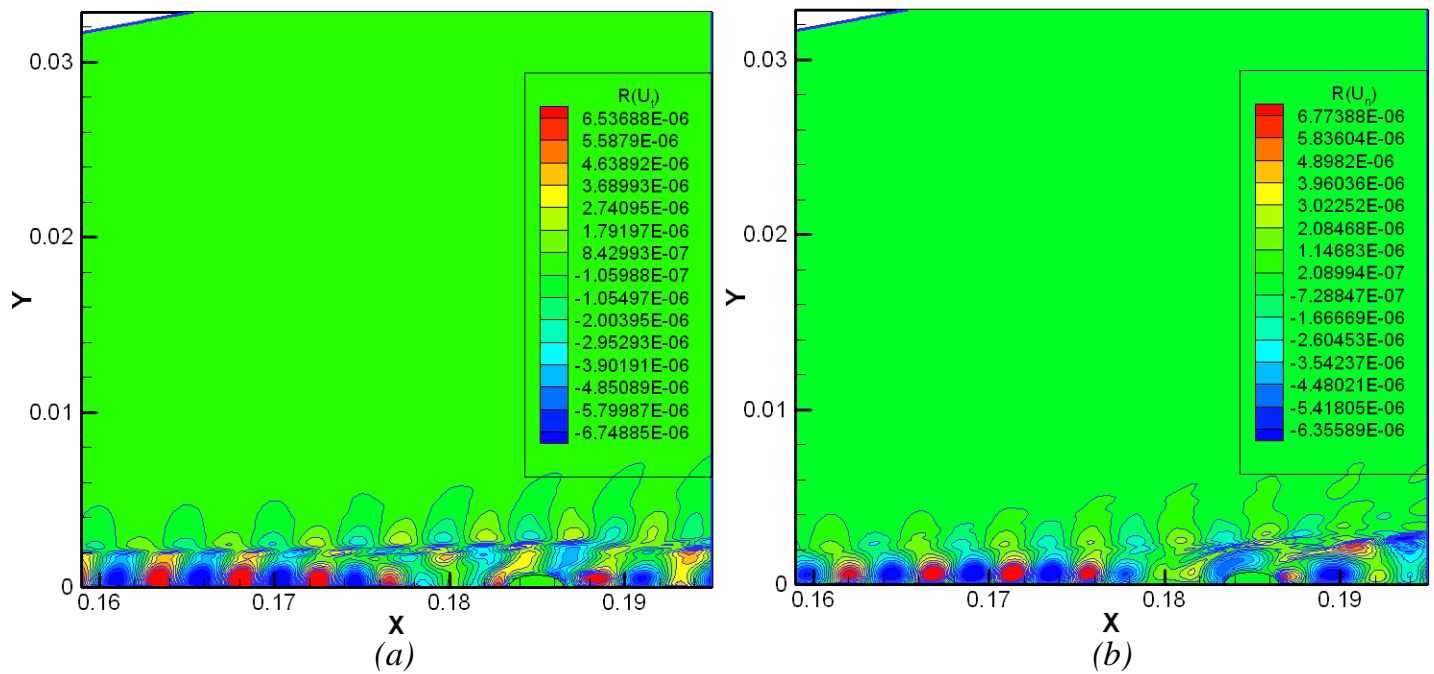

Figure 17. Contours for unsteady Mach 5.92 flow over a flat plate zone 8 with surface roughness (a), pressures (b), wall-normall velocity. 

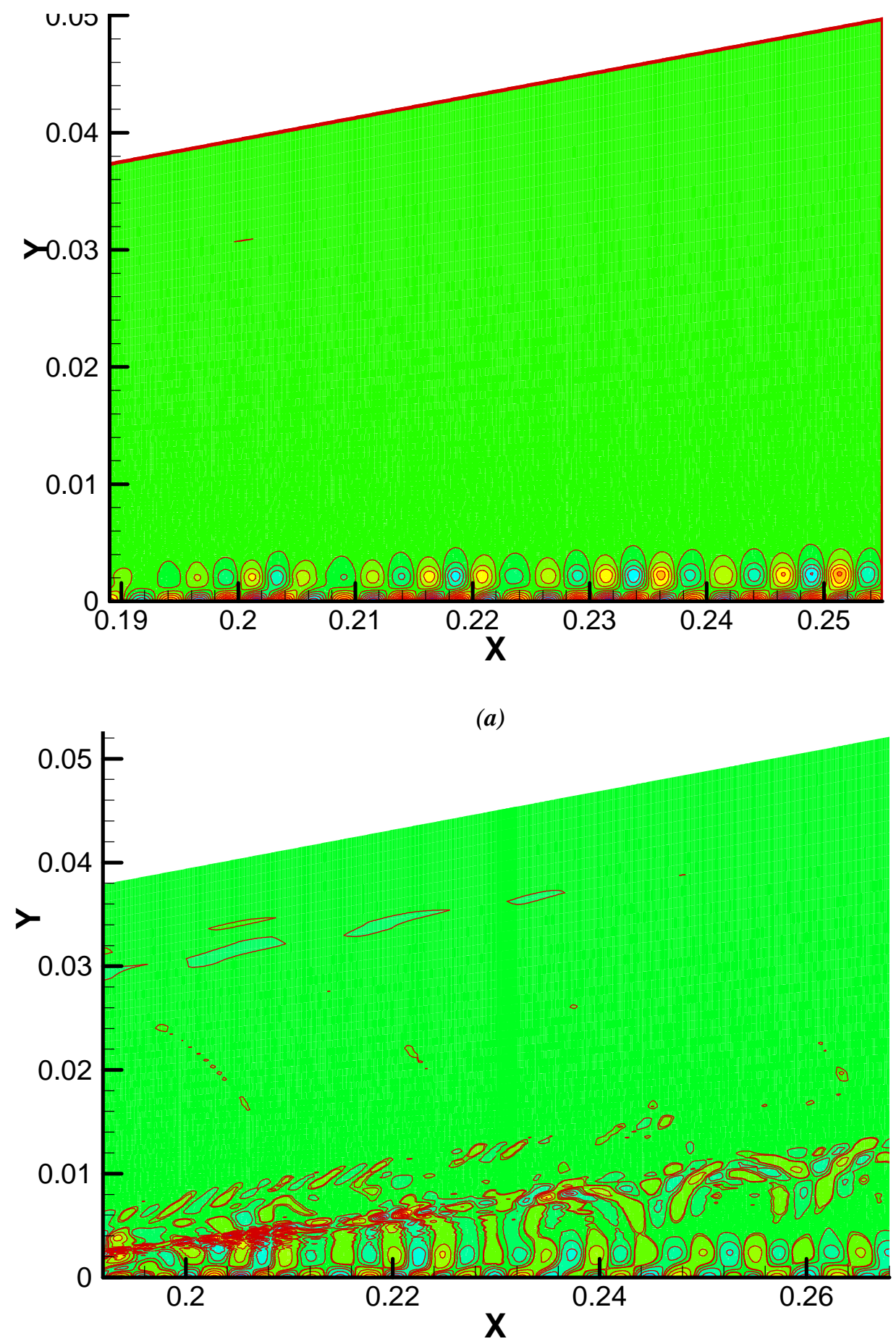

(b)

Figure 18. Pressure contours for unsteady Mach 5.92 flow over a flat plate zone 9 and zone 10 (a), without surface roughness (b), with surface roughness 


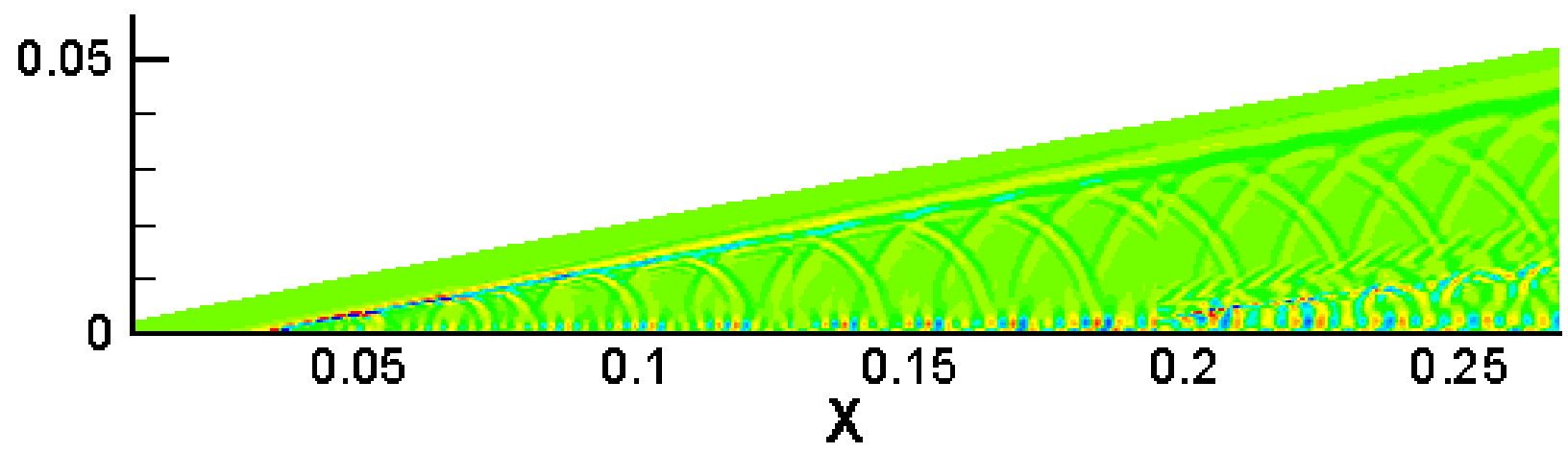

Figure 19. Pressure disturbance contour for unsteady flow field with external disturbance from zone 3 to zone 10.

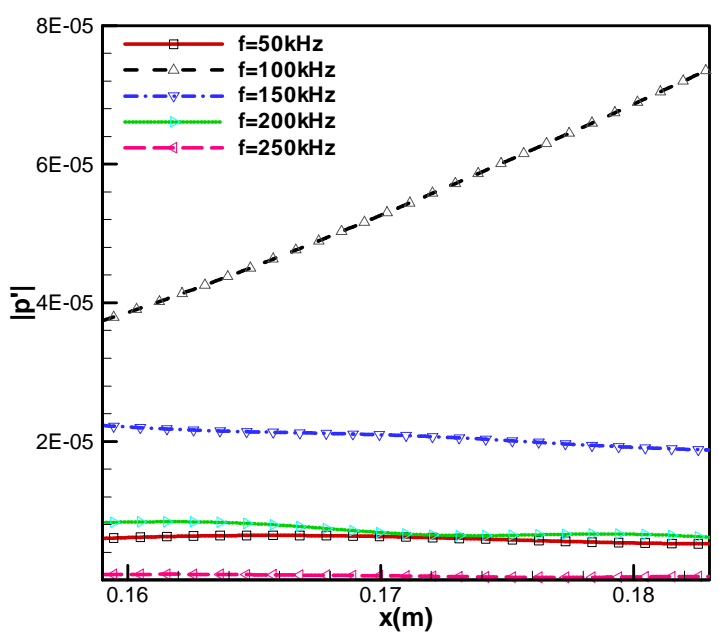

(a)

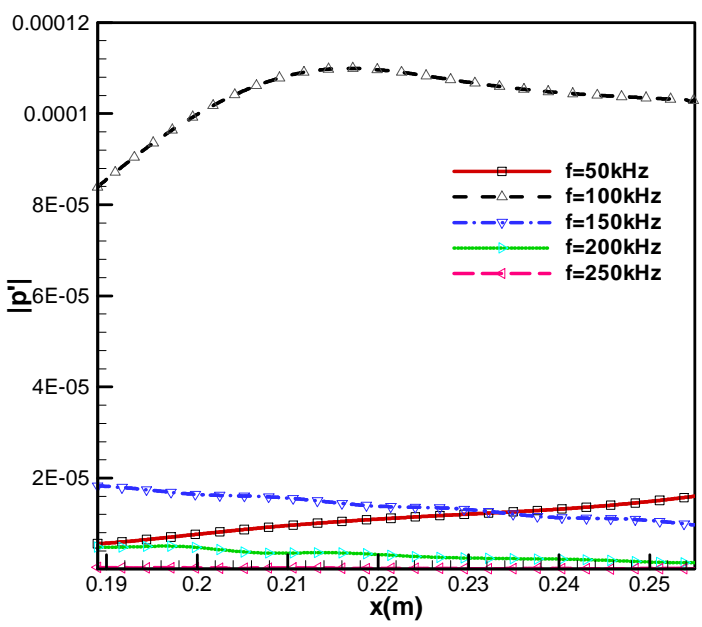

(c)

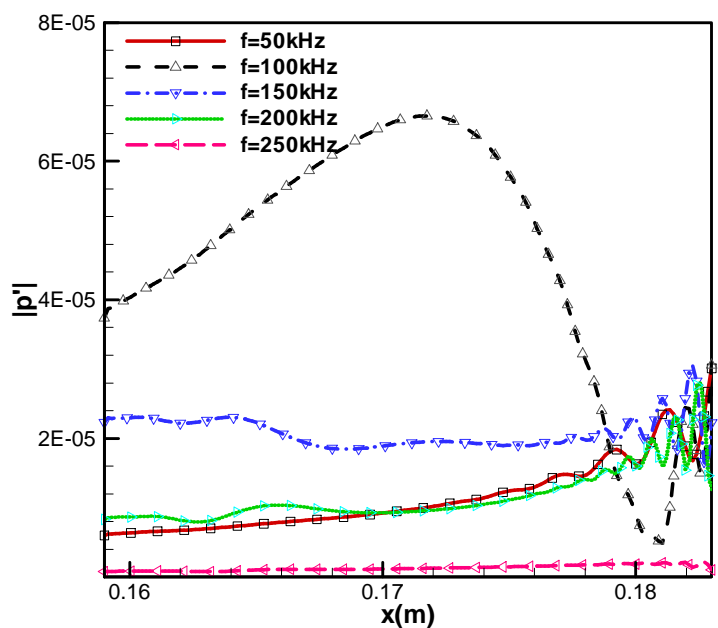

(b)

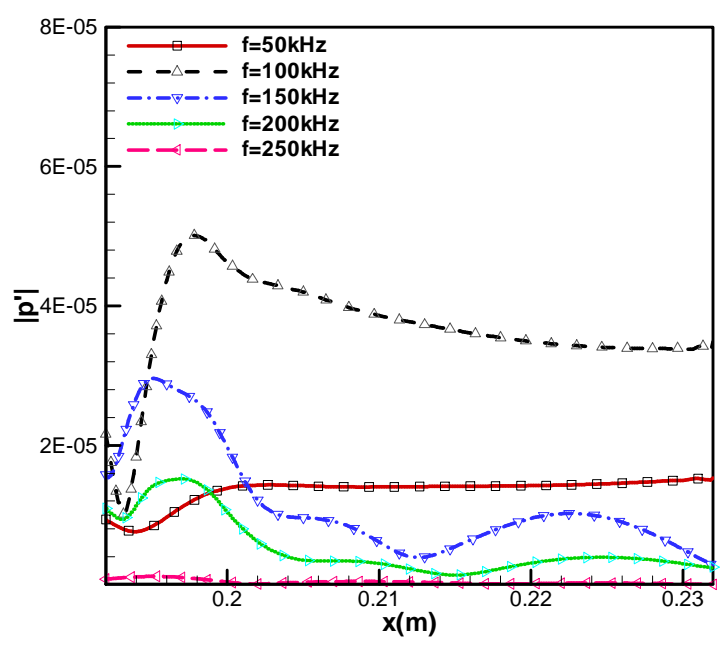

(d)

Figure 20. Maximum magnitude of streamwise pressure disturbance with different frequency after FFT for Mach 5.92 flow over flat-plate at zone $8(a)$, range $0.159 \mathrm{~m}-0.184 \mathrm{~m}$ for case without roughness $(b)$, range $0.159 \mathrm{~m}-0.184 \mathrm{~m}$ 
for case with roughness (c), range 0.186m-0.234m for case without roughness (d), range 0.186m-0.234m for case without roughness.

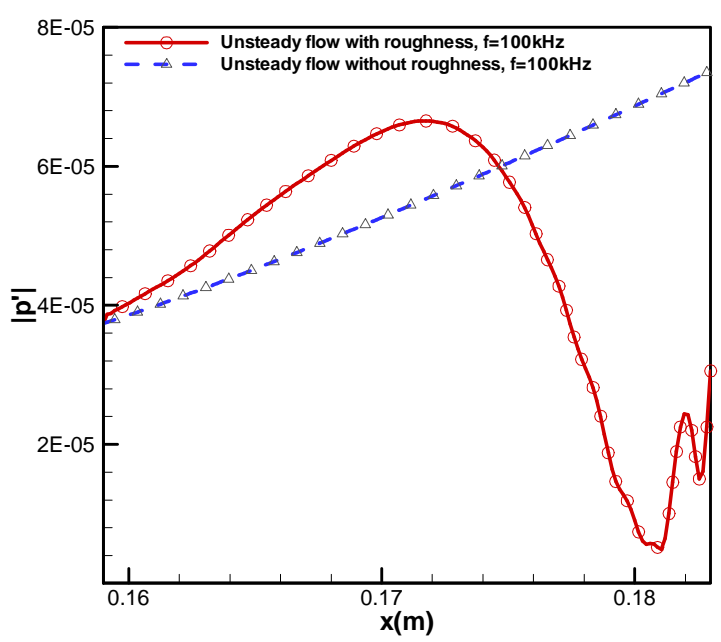

(a)

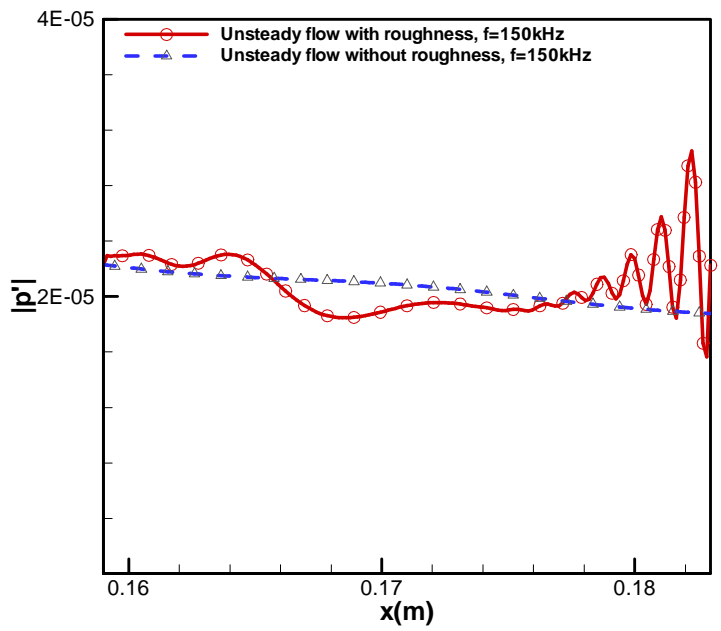

(c)

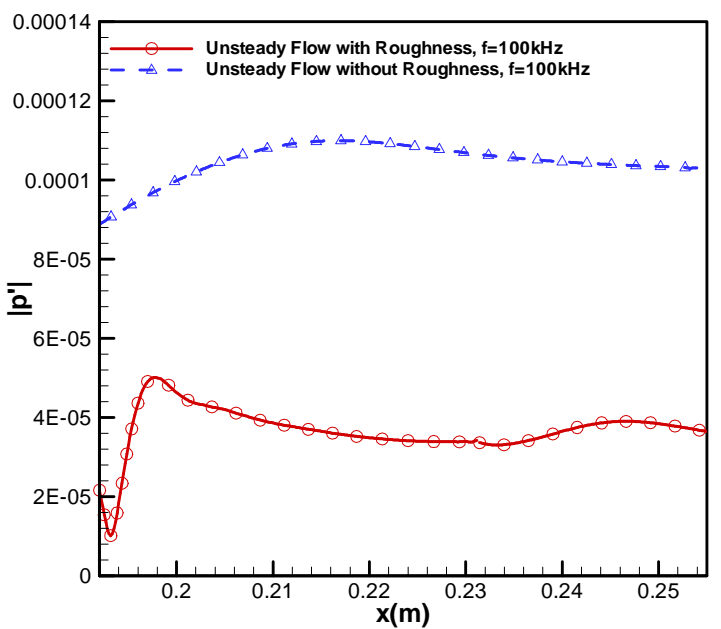

(b)

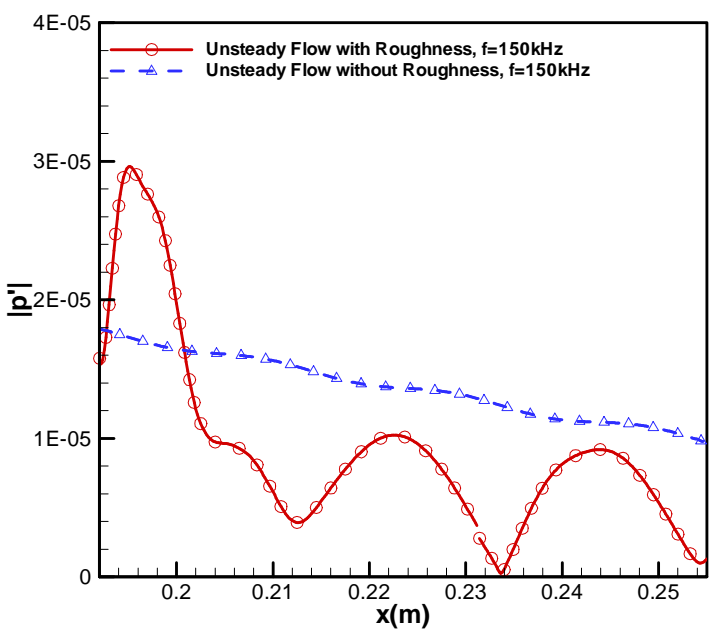

(d)

Figure 21. Maximum magnitude of streamwise velocity disturbance with different frequency after FFT for Mach 5.92 flow over flat-plate at zone 8 (a), $f=100 \mathrm{kHz}$,before roughness (b), $f=100 \mathrm{kHz}$,after roughness (c), $f=150 \mathrm{kHz}$,before roughness $(d), f=150 \mathrm{kHz}$, after roughness. 


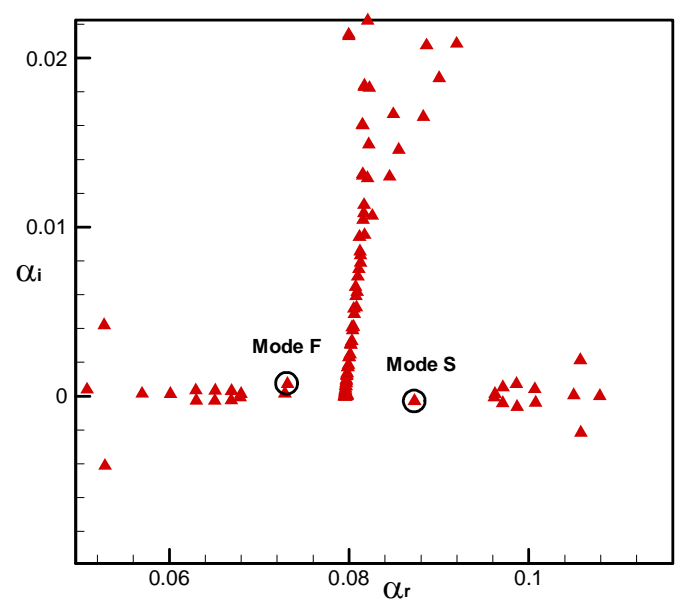

(a)

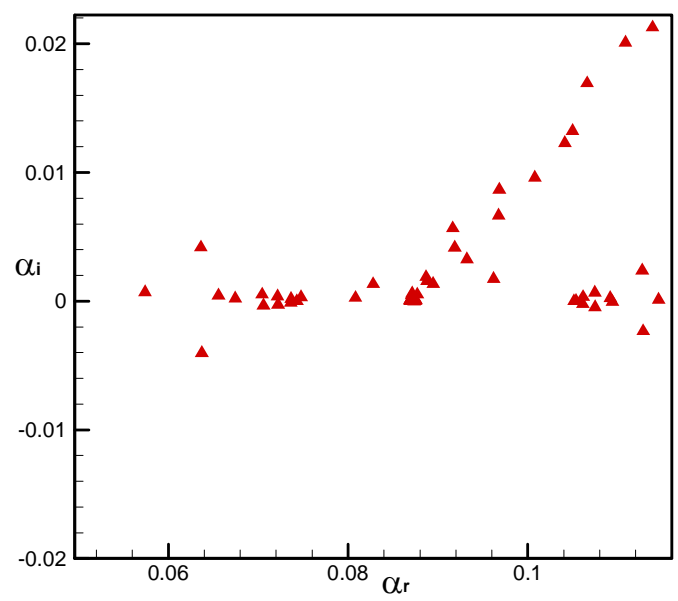

(c)

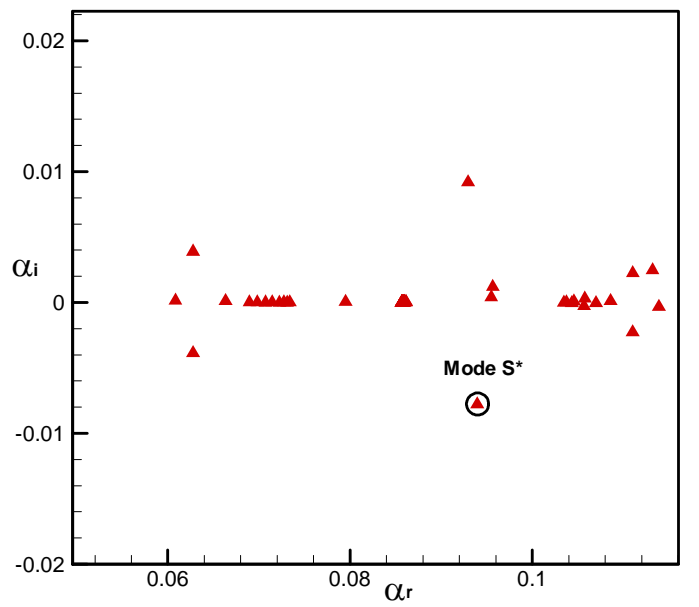

(b)

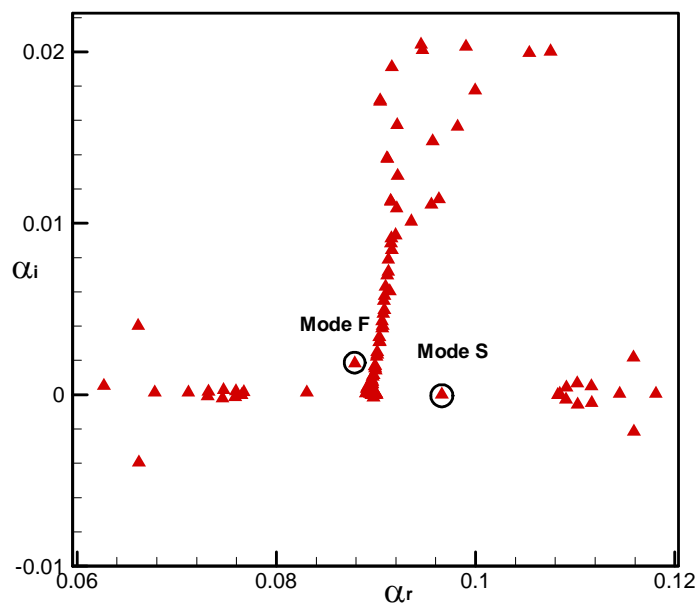

(d)

Figure 22. Discrete and continuous spectrum of $\alpha$ after applying LST to flow intersection (a) in upstream of roughness $x=0.1620 \mathrm{~m}(b)$, in close upstream roughness $x=0.1740 \mathrm{~m}(c)$, in close downstream of roughness $x=0.1890 \mathrm{~m},(d)$, in downstream of roughness $x=0.1980 \mathrm{~m}$. 


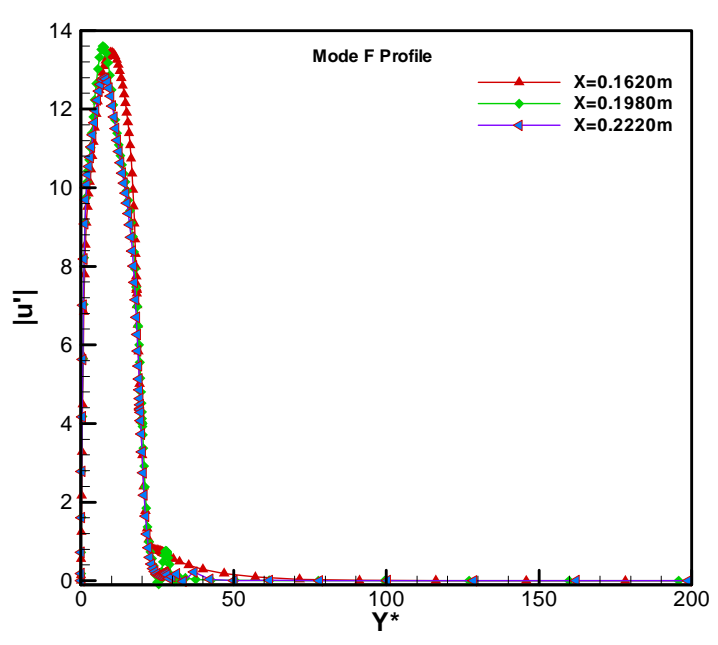

(a)

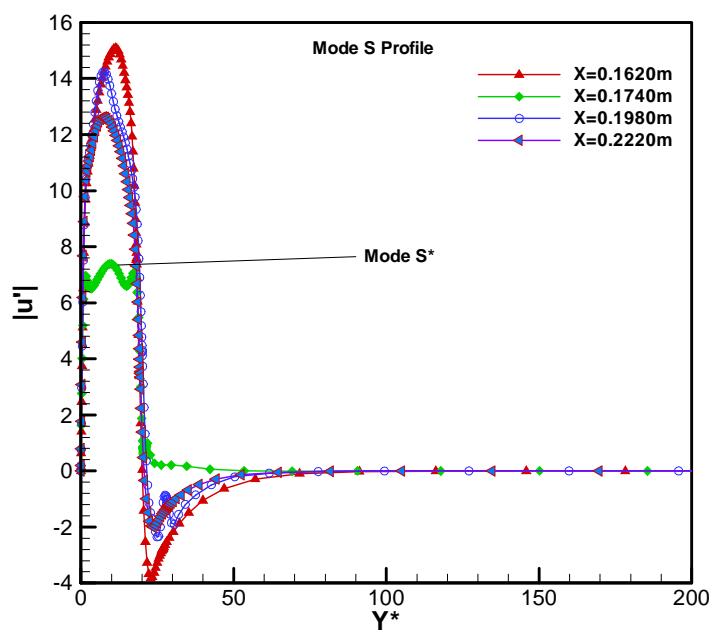

(c)

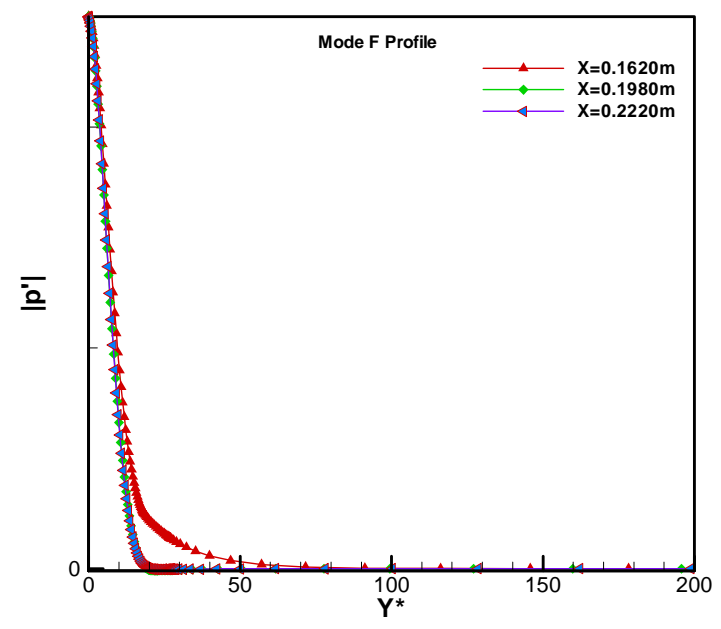

(b)

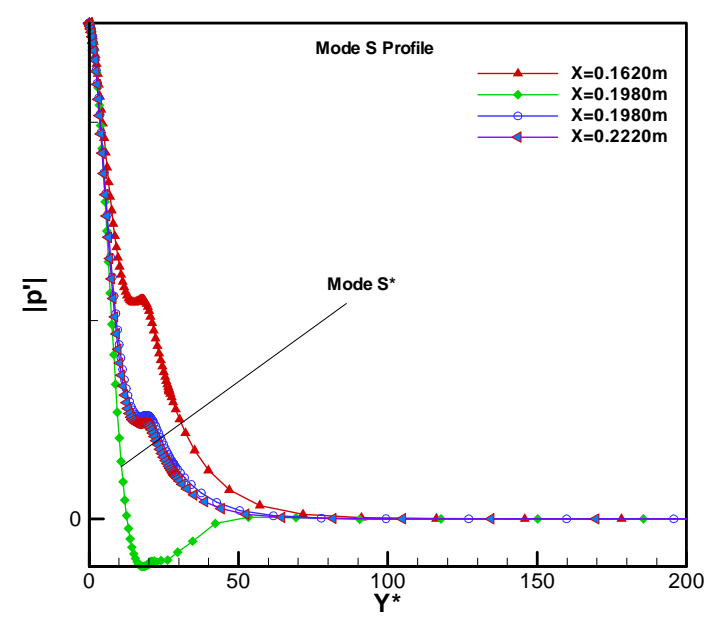

(d)

Figure 23. Mode profile of after applying LST to flow intersection at various location (a) Mode F profile for velocity (b), Mode F profile for pressure (c), Mode S profile for velocity, (d), Mode S profile for pressure. The amplitude of disturbances is nondimensionlized by its corresponding value on the surface of flat plate, e.g. $u^{\prime}(y)=\tilde{u}(y) / \tilde{u}(0)$ 


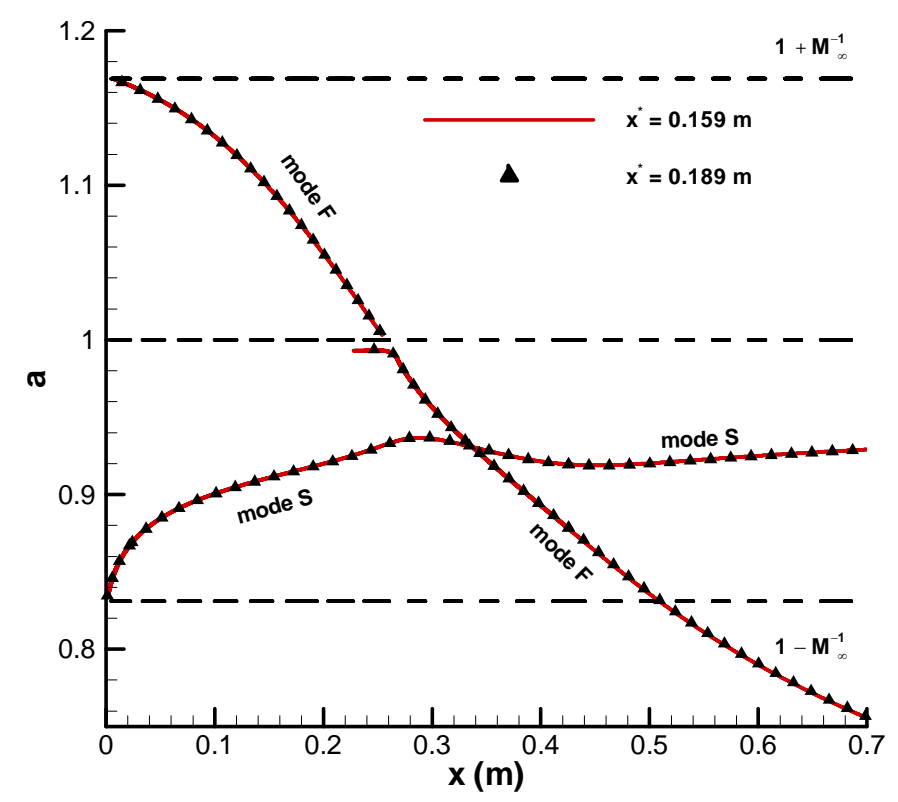

(a)

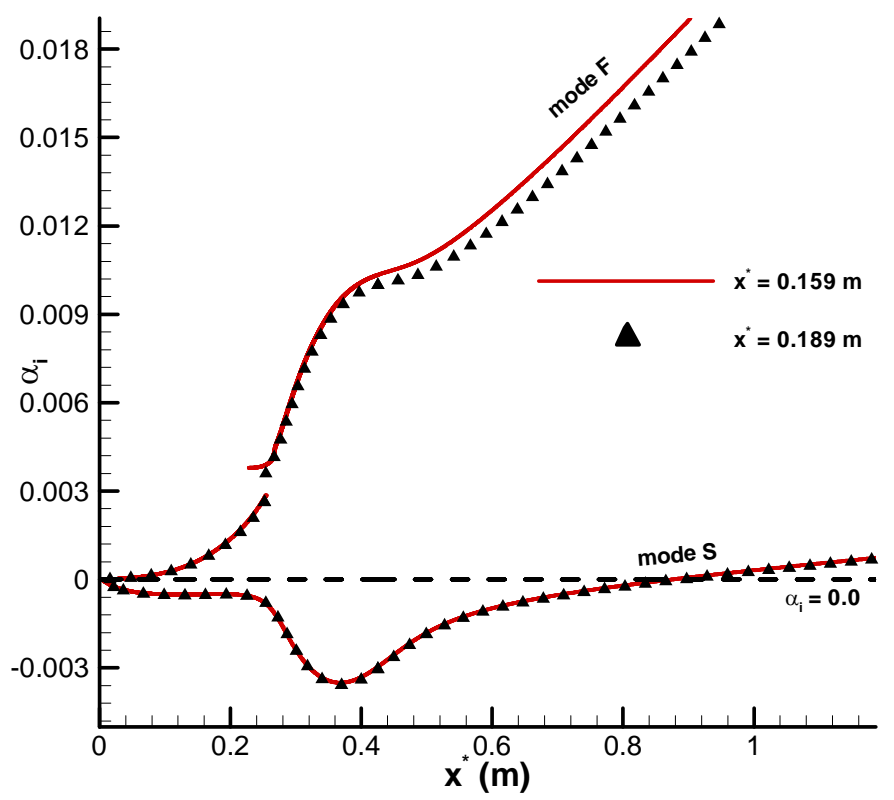

(b)

Figure 24. Eigenvalue of Mode $S$ and Mode F variation vs $x(m)$ for case without roughness via LST analysis. (a) wave speed $a=\frac{F \operatorname{Re}}{\alpha_{r}}(b)$,growth rate $\alpha_{i}$. Negative $\alpha_{i}$ imply Mode $S$ is unstable from wang et al. [44]. 


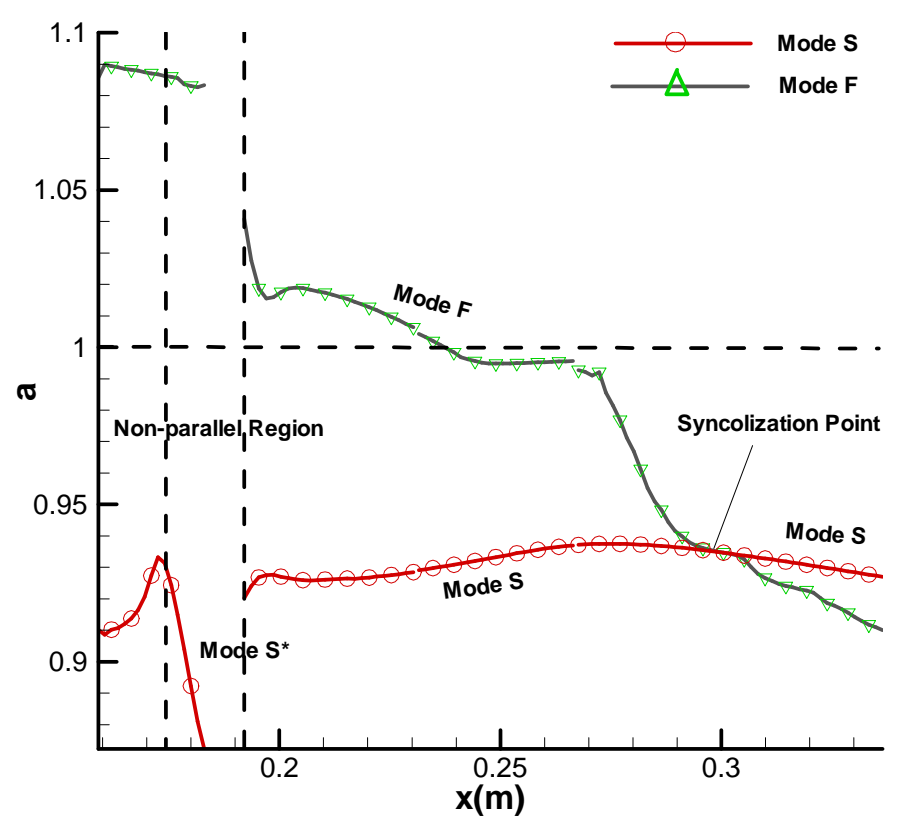

(a)

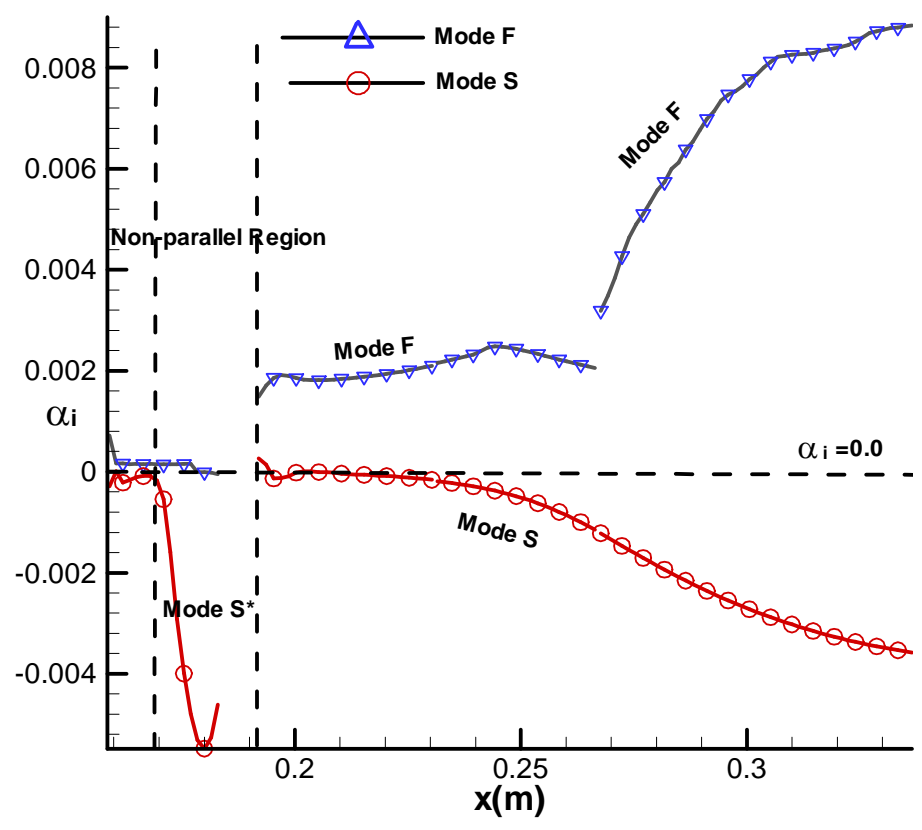

(b)

Figure 25. Eigenvalue of Mode $S$ and Mode F variation vs $x(m)$ for case with roughness via LST analysis. (a) wave speed $a=\frac{F \mathrm{Re}}{\alpha_{r}}(b)$,growth rate $\alpha_{i}$. Negative $\alpha_{i}$ imply Mode $S$ is unstable. The dash lines enclose region where the non-parallel flow assumption is violated. 